\title{
Four-Point Functions with a Twist
}

\author{
Till BARgheER \\ DESY Theory Group, DESY Hamburg, \\ Notkestraße 85, D-22603 Hamburg, Germany \\ till.bargheer@desy.de
}

\begin{abstract}
We study the OPE of correlation functions of local operators in planar $\mathcal{N}=4$ super Yang-Mills theory. The considered operators have an explicit spacetime dependence that is defined by twisting the translation generators with certain R-symmetry generators. We restrict to operators that carry a small number of excitations above the twisted BMN vacuum. The OPE limit of the four-point correlator is dominated by internal states with few magnons on top of the vacuum. The twisting directly couples all spacetime dependence of the correlator to these magnons. We analyze the OPE in detail, and single out the extremal states that have to cancel all double-trace contributions.
\end{abstract}




\section{Contents}

1 Introduction $\quad 1$

2 Twisted States $\quad 2$

3 Two-Point and Three-Point Correlators $\quad 6$

4 Four-Point Correlators $\quad 10$

5 OPE Analysis $\quad 13$

6 Conclusions $\quad 19$

$\begin{array}{ll}\text { A Details on Tensors } & 21\end{array}$

\section{Introduction}

Solving planar $\mathcal{N}=4$ super Yang-Mills theory (sYM) at any value of its coupling constant continues to be an important goal in mathematical physics. This most symmetric four-dimensional gauge theory serves as a key towards understanding general properties of interacting gauge theory, AdS/CFT duality, and quantum gravity. In probing the theory at ever deeper levels, we have witnessed extraordinary progress due to the emergence of integrability [1]. By now, the spectrum of single-trace operators is virtually solved, for any value of the coupling constant [2]. In the past few years, also three-point functions of local operators, as well as scattering amplitudes have come into focus. For both types of observables, essential proposals for finite-coupling descriptions have been made [3,4], and highly non-trivial implications could be derived from these proposals [5]. At this point, it appears not unlikely that all correlation functions will eventually be computable at any value of the coupling, at least in the planar limit.

In this paper, we focus on four-point correlation functions of local gauge-invariant operators. While two- and three-point functions in principle determine all higher-point functions via the conformal operator product expansion, actually resumming the expansion to recover explicit higher-point functions is very difficult in practice. Conversely, four-point (and higher-point) functions contain a wealth of information on structure constants and scaling dimensions, and are thus very interesting physical objects in their own right. It therefore remains desirable to devise efficient methods for computing higher-point correlators directly. Here, our primary goal will be to find the right language and variables for making use of the integrable structure for general correlation functions.

A key point of the integrability-based solution to the spectral problem is the organization of local operators in terms of excitations above a "vacuum" BPS operator $\operatorname{tr}\left[Z^{J}\right]$, where $Z$ is a complex scalar. The spectrum of anomalous scaling dimensions is then encoded in the eigenstates and eigenvalues of the dilatation generator, which are governed by the Beisert scattering matrix among the set of excitations (scalars, fermions, and covariant derivatives) above $\operatorname{the} \operatorname{tr}\left[Z^{J}\right]$ vacuum $[6$. In particular, the scattering matrix is, up to its overall phase, completely constrained by the $\mathfrak{p} \mathfrak{s u}(2 \mid 2)^{2}$ subalgebra of the superconformal symmetry that preserves the vacuum.

For higher-point functions, it is again desirable to consider operators that preserve as much symmetry as possible. The familiar BPS operators $\operatorname{tr}\left[Z^{J}\right]$ suggest themselves. But when 
considering more than two insertions of such operators, most of the symmetry is broken, because the $\mathfrak{p s u}(2 \mid 2)^{2}$ symmetries of the individual insertions are not aligned. The situation can be improved by considering slightly generalized BPS operators, which are related to $\operatorname{tr}\left[Z^{J}\right]$ by an internal rotation. Namely, consider $\operatorname{tr}\left[\phi^{J}\right]$, where $\phi=X \cdot \Phi$ and $X \in \mathbb{C}^{6}, X^{2}=0$ specifies a complex, lightlike direction in the internal space of scalar fields $\Phi^{I}, I=1, \ldots, 6$. By judiciously choosing the vector $X$ as a function of the spacetime coordinate, a substantial part of the superconformal symmetry can be preserved. This has been noted before [7,8]. Namely, after choosing a vector $X$ at the spacetime origin $x=0$, define $X(x)$ throughout spacetime via a twisted translation generator $\check{\mathfrak{P}} \sim \mathfrak{P}-\mathfrak{R}$ that is a combination of the conventional spacetime translation $\mathfrak{P}$ and an internal rotation $\mathfrak{R}$. This twisted translation is associated to a whole twisted conformal symmetry algebra $\widehat{\mathfrak{s o}}(2,4)$, which is a diagonal combination of the original conformal algebra $\mathfrak{s o}(2,4)$ and the internal $\mathfrak{s o}(6)$ R-symmetry. The twisted fields were first considered in the context of topological twistings of $\mathcal{N}=4$ super Yang-Mills theory [7]. Later, correlation functions of single-trace operators of a single twisted complex scalar $\phi$ were considered [8], and found to (i) have trivial spacetime dependence, and (ii) be protected from quantum corrections. These properties make such operators good candidates for an integrability-based description in terms of excitations on top of vacuum operators $\operatorname{tr}[\phi \ldots \phi]$. In fact, the hexagon form factor proposal for three-point functions [4] relies on an excitation picture around vacuum operators of this type.

In this paper, we consider four-point functions of twisted single-trace operators with few excitations at the one-loop level. For the time being, the analysis is restricted to zero-momentum excitations. While this means that the four operators are still half-BPS, their four-point functions are not protected. We extract the one-loop correlation functions of such operators from [9], and analyze their OPE decomposition. In the double-coincidence limit $\left|x_{12}\right|,\left|x_{34}\right| \ll\left|x_{23}\right|$, the one-loop correlator displays a logarithmic singularity, which is generated by the anomalous dimensions of the internal states in the OPE. We show how this leading term is captured by states with a minimal number of excitations on top of the twisted vacuum. For "extremal" OPE contributions (which stem from internal states with the maximal R-charge compatible with the external operators), it is known that there is a $1 / N_{\mathrm{c}}^{2}$ mixing with double-trace operators. We find that the contribution of double-trace operators can be neglected by simply projecting out specific extremal states in the OPE.

Note: While this work was being completed, I learned of the very interesting parallel paper [10], which also discusses the OPE of four BPS operators from an integrability perspective. While this work considers operators of any charge, [10] focuses on operators with large charges and the relation to the hexagon form factor approach.

\section{Twisted States}

We start by reviewing essential parts of [7], [8], and [11]. Consider a general complex linear combination $X \cdot \Phi=X_{I} \Phi^{I}$ of the six real scalar fields $\Phi^{I}$. The propagator for two such fields equals (neglecting color factors)

$$
\left\langle X_{1} \cdot \Phi\left(x_{1}\right) X_{2} \cdot \Phi\left(x_{2}\right)\right\rangle=\frac{X_{1} \cdot X_{2}}{(2 \pi)^{2} x_{12}^{2}} .
$$

Correlation functions of single-trace operators are difficult to compute in general, and have a complicated dependence on the spacetime coordinates as well as the coupling constant, unless they obey extra relations due to supersymmetry. A prominent case are half-BPS chiral 
primary operators $\operatorname{tr}\left[(X \cdot \Phi)^{J}\right]$ with $X^{2}=0$. Every such operator preserves 24 supersymmetries. Therefore any two such operators share 16 supersymmetries, and any three such operators share 8 supersymmetries. This is the reason why two-point and three-point functions of such half-BPS operators are protected from quantum corrections. The same is generically not true for correlation functions of four or more such operators, which generically do not share any supersymmetry. However, making the judicious, spacetime-dependent choice

$$
\begin{gathered}
X=X^{\phi}\left(x^{\mu}\right) \equiv \sqrt{2}\left(i x^{\mu}, \frac{1}{2}\left(1+x^{2}\right), \frac{i}{2}\left(1-x^{2}\right)\right) \\
\phi(x) \equiv X^{\phi}(x) \cdot \Phi(x)
\end{gathered}
$$

has two important consequences. Firstly, $X^{\phi}\left(x_{1}\right) \cdot X^{\phi}\left(x_{2}\right)=x_{12}^{2}$, therefore the free propagator of two fields $\phi$ becomes constant,

$$
\left\langle\phi\left(x_{1}\right) \phi\left(x_{2}\right)\right\rangle=\frac{1}{(2 \pi)^{2}} .
$$

Hence, in the free field theory, correlation functions of any number of operators

$$
\mathcal{Q}^{J}(x) \equiv \operatorname{tr}\left[\phi(x)^{J}\right]
$$

are completely spacetime-independent and just evaluate to constants. Secondly, all correlation functions

$$
\left\langle\mathcal{Q}^{J_{1}}\left(x_{1}\right) \ldots \mathcal{Q}^{J_{n}}\left(x_{n}\right)\right\rangle
$$

are protected from quantum corrections, for any number $n$ of operator insertions. The reason is that all operators $\mathcal{Q}^{J}(x)$ share two universal supercharges $\mathfrak{Q}^{ \pm}$, and the perturbative action of $\mathcal{N}=4 \mathrm{sYM}$ is $\mathfrak{Q}^{ \pm}$-exact $\left.[7,8] .1\right]$ We will refer to the operators $(2.5)$ as vacuum operators.

The field $\phi(x)$ can be defined via a translation that is twisted with an appropriate R-symmetry generator. In fact, the field $(2.3)$ was first considered in the context of topological twists of $\mathcal{N}=4 \mathrm{sYM}|7|$. The twisted translation is associated with a whole twisted conformal symmetry algebra $\widehat{\mathfrak{s o}}(2,4)$, which is a diagonal combination of the original conformal algebra $\mathfrak{s o}(2,4)$ and the internal $\mathfrak{s o}(6)$ R-symmetry. Denoting the generators of the original conformal symmetry by $\left\{\mathfrak{P}_{\mu}, \mathfrak{K}_{\mu}, \mathfrak{L}_{\mu \nu}, \mathfrak{D}\right\}$, the twisted conformal symmetry generators read ${ }^{2}$

$$
\begin{aligned}
\hat{\mathfrak{P}}_{\mu} & =\mathfrak{P}_{\mu}+\mathfrak{R}_{\mu-}, & \hat{\mathfrak{L}}_{\mu \nu} & =\mathfrak{L}_{\mu \nu}+\mathfrak{R}_{\mu \nu}, \\
\hat{\mathfrak{K}}_{\mu} & =\mathfrak{K}_{\mu}+\mathfrak{R}_{\mu+}, & \hat{\mathfrak{D}} & =\mathfrak{D}+\mathfrak{R} .
\end{aligned}
$$

Here, the R-symmetry algebra has been split into an $\mathfrak{s o}(4)$ with generators $\mathfrak{R}_{\mu \nu}$ and a remainder with generators $\mathfrak{R}_{\mu \pm} \equiv-i \mathfrak{R}_{\mu 5} \pm \mathfrak{R}_{\mu 6}$, and $\mathfrak{R} \equiv-i \mathfrak{R}_{56}$. Starting with $\phi(0)=\left(\Phi^{5}+i \Phi^{6}\right) / \sqrt{2} \equiv Z$ at the origin, $\phi(x)$ throughout spacetime is obtained by

$$
\phi(x) \equiv \exp \left(x^{\mu}\left(\mathfrak{P}_{\mu}-\mathfrak{R}_{\mu-}\right)\right) \cdot Z(0)=\exp \left(-x^{\mu} \mathfrak{R}_{\mu-}\right) \cdot Z(x) .
$$

The splitting is chosen such that $\mathfrak{R}_{\mu+}$ annihilates $\phi(0)=Z$ at the origin, and $\mathfrak{R}$ measures the R-charge along the direction of $Z$,

$$
\mathfrak{R} \cdot Z=+Z, \quad \mathfrak{R} \cdot \bar{Z}=-\bar{Z}, \quad\left[\mathfrak{R}, \mathfrak{R}_{\mu \pm}\right]= \pm \mathfrak{R}_{\mu \pm}
$$

\footnotetext{
${ }^{1}$ See also $[12$ for an OPE-based argument that does not refer to the Lagrangian.

${ }^{2}$ A similar twisting was used in $\left.\mid 13\right]$ to construct a chiral algebra of operators living in a two-dimensional hyperplane of the four-dimensional Minkowski spacetime. The construction of $[13$ involves passing to the cohomology of a suitable supercharge, upon which all operators transform trivially under a twisted conformal algebra. On the contrary, here we study fully four-dimensional operators that transform non-trivially under the twisted conformal symmetry.
} 
Note that $\mathfrak{R}_{\mu-}$ appears with opposite signs in $(\overline{2.7})$ and $(\overline{2.8})$. This is natural: The exponential in $(2.8)$ "undoes" the twisting defined by $(2.7)$, such that the twisted translation generator $\hat{\mathfrak{P}}_{\mu}$ simply acts as

$$
\hat{\mathfrak{P}}_{\mu} \phi=\partial_{\mu} \phi,
$$

where $\partial_{\mu}$ only acts on the fields $\Phi^{I}$, and not on the explicit spacetime coordinates $x$. Together with $\phi$, the remainder of the six scalars $\Phi^{I}$ naturally organizes into a multiplet $\left\{\phi, V_{\mu}, B\right\}$ of the twisted conformal symmetry, with

$$
\begin{aligned}
V_{\mu} & \equiv X^{V_{\mu}} \cdot \Phi, & X^{V_{\mu}} & \equiv \frac{1}{\sqrt{2}}\left(\partial_{\mu} X^{\phi}\right)=\left(i e_{\mu}, x_{\mu},-i x_{\mu}\right), \\
B & \equiv X^{B} \cdot \Phi, & X^{B} & \equiv \frac{1}{4 \sqrt{2}}\left(\partial^{2} X^{\phi}\right)=(0,0,0,0,1,-i) .
\end{aligned}
$$

Just as $\phi(x)$, the fields $V_{\mu}(x)$ and $B(x)$ throughout spacetime are obtained by the same twisted translation $(2.8)$ applied to $V_{\mu}(0)$ and $B(0)$ at the origin. The latter reduce to

$$
V_{\mu}(0)=i \Phi_{\mu}, \quad B(0)=\Phi_{5}-i \Phi_{6}=\sqrt{2} \bar{Z} .
$$

The twisted translation is defined such that, when acting on the twisted fields $\phi, V_{\mu}$, and $B$, the generators of the twisted conformal algebra $\widehat{\mathfrak{s o}}(2,4)$ take the standard realization

$$
\begin{aligned}
\hat{\mathfrak{P}}_{\mu} & =\partial_{\mu} \\
\hat{\mathfrak{L}}_{\mu \nu} & =\left(x_{\mu} \partial_{\nu}-x_{\nu} \partial_{\mu}\right)+\hat{\Sigma}_{\mu \nu}, \\
\hat{\mathfrak{D}} & =x^{\mu} \partial_{\mu}+\hat{\Delta} \\
\hat{\mathfrak{K}}_{\mu} & =2 x_{\mu} x \cdot \partial-x^{2} \partial_{\mu}-2 x_{\mu} \hat{\Delta}+2 x^{\nu} \Sigma_{\mu \nu}+\hat{\kappa}_{\mu},
\end{aligned}
$$

where the derivatives only act on the fields $\Phi^{I}$, and not on the explicit coordinates $x^{\mu}$ in the definition of the fields $\phi, V_{\mu}$, and $B$. The generators $\hat{\Delta}, \hat{\Sigma}_{\mu \nu}$ and $\hat{\kappa}_{\mu}$ specify the transformation properties of the fields at the origin,

$$
\begin{aligned}
& {[\hat{\Delta}, \phi]=0, \quad\left[\hat{\Sigma}_{\mu \nu}, \phi\right]=0, \quad\left[\hat{\kappa}_{\mu}, \phi\right]=0,} \\
& {\left[\hat{\Delta}, V_{\rho}\right]=V_{\rho}, \quad\left[\hat{\Sigma}_{\mu \nu}, V_{\rho}\right]=\eta_{\rho \mu} V_{\nu}-\eta_{\rho \nu} V_{\mu}, \quad\left[\hat{\kappa}_{\mu}, V_{\rho}\right]=-\eta_{\mu \rho} \sqrt{2} \phi \text {, }} \\
& {[\hat{\Delta}, B]=2 B, \quad\left[\hat{\Sigma}_{\mu \nu}, B\right]=0, \quad\left[\hat{\kappa}_{\mu}, B\right]=-2 V_{\mu} \text {. }}
\end{aligned}
$$

That is $\phi$ is a scalar with dimension zero, $V_{\mu}$ is a vector with dimension one, and $B$ is a scalar with dimension two under the twisted conformal symmetry. More generally, by construction, the twisted scaling dimension of any operator is the sum of the untwisted scaling dimenion and the $\mathrm{R}$-charge in the $Z$ direction. What is nonstandard is that the special conformal generator $\hat{\mathfrak{K}}$ acts non-trivially at the origin, even though the fields $V_{\rho}$ and $B$ are not conformal descendants. ${ }^{3}$ Due to the non-trivial action of $\hat{\kappa}$, the representation of the little group (with generators $\hat{\Sigma}_{\mu \nu}$, $\hat{\Delta}$, and $\hat{\kappa}_{\mu}$ ) is not further reducible, even though $\phi, V_{\mu}$, and $B$ have different scaling dimensions. This affects the conformal Ward identities for correlation functions, and hence correlators of twisted fields generically do not have the standard form (that follows from the Ward identities for $\hat{\kappa}_{\mu}=0$ ). In particular, operators with different twisted dimensions may have non-vanishing two-point functions.

The twisting is explained in detail in $[7,8]$. Here, we only note that under the twisted conformal symmetry, the fermion fields organize into vectors $\psi_{\mu}^{(1)}, \widetilde{\psi}_{\mu}^{(1)}$, two-form fields $\chi_{\mu \nu}^{ \pm,(2)}$,

\footnotetext{
${ }^{3}$ In the classification of representations of the conformal algebra by Mack and Salam [14], the representation (2.15) belongs to class $\mathrm{Ib}\left(\kappa_{\mu} \neq 0\right.$ but nilpotent).
} 
and scalars $\eta^{(2)}, \tilde{\eta}^{(2)}$, where the superscript numbers denote the twisted scaling dimensions. The gauge fields are R-symmetry singlets and thus not affected by the twisting. The twisted matter fields $\left\{\phi, V_{\mu}, B, \psi_{\mu}, \tilde{\psi}_{\mu}, \chi_{\mu \nu}^{ \pm}, \eta, \tilde{\eta}\right\}$ transform covariantly under fifteen combinations $\mathfrak{Q}_{\mu}, \mathfrak{S}_{\mu}, \mathfrak{Q}_{\mu \nu}$, and $\mathfrak{Q}_{\mathrm{D}}$ of the fermionic generators of $\mathfrak{p s u}(2,2 \mid 4)$. Commuting the latter with the singlets $\mathfrak{Q}^{ \pm}$ that preserve the vacuum field $\phi$ yields the twisted conformal algebra,

$$
\begin{array}{rlrl}
{\left[\mathfrak{Q}^{ \pm}, \mathfrak{Q}_{\mu}\right]} & =\hat{\mathfrak{P}}_{\mu}, & & {\left[\mathfrak{Q}^{ \pm}, \mathfrak{Q}_{\mu \nu}\right]=\hat{\mathfrak{L}}_{\mu \nu},} \\
{\left[\mathfrak{Q}^{ \pm}, \mathfrak{S}_{\mu}\right]=\hat{\mathfrak{K}}_{\mu},} & {\left[\mathfrak{Q}^{ \pm}, \mathfrak{Q}_{\mathrm{D}}\right]=\hat{\mathfrak{D}} .}
\end{array}
$$

The fermionic generators $\left\{\mathfrak{Q}_{\mu}, \mathfrak{S}_{\mu}, \mathfrak{Q}_{\mu \nu}, \mathfrak{Q}_{\mathrm{D}}\right\}$ and their bosonic counterparts $\left\{\hat{\mathfrak{P}}_{\mu}, \hat{\mathfrak{K}}_{\mu}, \hat{\mathfrak{L}}_{\mu \nu}, \hat{\mathfrak{D}}\right\}$ combine into the strange superalgebra $\mathfrak{q}(3)[15$.

Besides the vacuum operators $\mathcal{Q}^{J}$, we will also consider operators with zero-momentum excitations (R-symmetry descendants of the vacuum)

$$
\mathcal{Q}_{\mu}^{J}=\operatorname{tr}\left[V_{\mu} \phi^{J-1}\right], \quad \mathcal{Q}_{(\mu \nu)}^{J}=\sum_{j=0}^{J-2} \operatorname{tr}\left[V_{(\mu} \phi^{j} V_{\nu)} \phi^{J-2-j}\right], \quad \ldots
$$

and two-magnon operators with non-zero momenta

$$
\begin{aligned}
\mathcal{O}_{p}^{J} & =\frac{1}{\sqrt{J+3}}\left[\frac{1}{2} \sum_{j=0}^{J} \cos (p(j+3 / 2)) \operatorname{tr}\left[V_{\mu} \phi^{j} V^{\mu} \phi^{J-j}\right]+\sqrt{2} \cos (p / 2) \operatorname{tr}\left[B \phi^{J+1}\right]\right], \\
\mathcal{O}_{p,(\mu \nu)}^{J, V V} & =\frac{1}{\sqrt{J+3}} \sum_{j=0}^{J+2} \cos (p(j+1 / 2)) \operatorname{tr}\left[V_{(\mu} \phi^{j} V_{\nu)} \phi^{J+2-j}\right], \\
\mathcal{O}_{p}^{J, \partial \partial} & =\frac{1}{\sqrt{J+3}} \frac{1}{4} \sum_{j=0}^{J+2} \cos (p(j+1 / 2)) \operatorname{tr}\left[\phi_{, \mu} \phi^{j} \phi^{, \mu} \phi^{J+2-j}\right]+\ldots, \\
\mathcal{O}_{p,(\mu \nu)}^{J, \partial \partial} & =\frac{1}{\sqrt{J+3}}\left[\frac{1}{2} \sum_{j=0}^{J} \cos (p(j+3 / 2)) \operatorname{tr}\left[\phi_{,(\mu} \phi^{j} \phi_{, \nu)} \phi^{J-j}\right]+\frac{1}{2} \cos (p / 2) \operatorname{tr}\left[\phi,(\mu \nu) \phi^{J+1}\right]\right], \\
\mathcal{O}_{p, \mu \nu}^{J, V \partial, 1} & =\frac{-i}{\sqrt{J+3}}\left[\frac{1}{\sqrt{2}} \sum_{j=0}^{J} \cos (p(j+3 / 2)) \operatorname{tr}\left[V_{\mu} \phi^{j} \phi_{, \nu} \phi^{J-j}\right]+\sqrt{2} \cos (p / 2) \operatorname{tr}\left[V_{\mu, \nu} \phi^{J+1}\right]+\ldots\right], \\
\mathcal{O}_{p, \mu \nu}^{J, V \partial, 2} & =\frac{-i}{\sqrt{J+3}} \frac{1}{\sqrt{2}} \sum_{j=0}^{J+2} \cos (p(j+1 / 2)) \operatorname{tr}\left[V_{\mu} \phi^{j} \phi_{, \nu} \phi^{J+2-j}\right]+\ldots
\end{aligned}
$$

Here, $\phi_{, \mu}=X^{\phi} \cdot D_{\mu} \Phi$ and $V_{\mu, \nu}=X^{V_{\mu}} \cdot D_{\nu} \Phi$ are the twisted combinations of covariant derivative fields $D_{\mu} \Phi^{I}$. These two-magnon states are simply twisted cousins of the two-magnon states listed in Appendix B of [11]. They all belong to a common superconformal multiplet whose primary is the operator $\mathcal{O}_{p}^{J}$, where $J$ labels the charge under the (untwisted) operator $\mathfrak{R}$ measured at the origin $x=0 . .^{4}$ The ellipses in (2.18) stand for terms with fermion fields and non-linear corrections which do not contribute to the free OPE coefficients. In the operators $(2.18)$, the two magnons carry opposite momenta $p$ and $-p$, with

$$
p \in \frac{2 \pi n}{J+3}, \quad 1 \leq n \leq\left\lfloor\frac{J+2}{2}\right\rfloor .
$$

Since the twist amounts to a (position-dependent) R-symmetry rotation, and the one-loop dilatation operator commutes with the R-symmetry, all eigenstates remain eigenstates, with

\footnotetext{
${ }^{4}$ The full supermultiplet contains further states, which however will not be relevant, since they are either fermionic or antisymmetric, and thus do not contribute to OPE's of operators $\mathcal{Q}^{J}, \mathcal{Q}_{\mu}^{J}$ considered below.
} 
unmodified eigenvalues, and all states with different eigenvalues remain orthogonal. ${ }^{5}$ In other words, the anomalous dimension is unaffected by the twisting. The two-magnon states therefore have twisted dimensions

$$
\hat{\Delta}(p)=2+\lambda \Delta_{1}(p)+\mathcal{O}\left(\lambda^{2}\right), \quad \Delta_{1}(p)=\frac{1}{\pi^{2}} \sin ^{2}(p / 2),
$$

with the familiar value $\Delta_{1}$ of the anomalous dimension.

While the operator $\mathcal{O}_{p}^{J}(x=0)$ carries (untwisted) $\mathfrak{R}$-charge $J$, the charges of the superdescendant operators $\mathcal{O}_{p, \ldots}^{J, \ldots}$ are shifted. Besides the charge $J$, we will sometimes refer to the "length" $L$, which equals the number of fields within a single-trace operator. The following table summarizes the $\mathfrak{R}$-charges (at $x=0$ ) and lengths of the various operators:

\begin{tabular}{cccccccccc}
\hline Operator: & $\mathcal{O}_{p}^{J}$ & $\mathcal{O}_{p,(\mu \nu)}^{J, V V}$ & $\mathcal{O}_{p}^{J, \partial \partial}$ & $\mathcal{O}_{p,(\mu \nu)}^{J, \partial \partial}$ & $\mathcal{O}_{p, \mu \nu}^{J, V \partial, 1}$ & $\mathcal{O}_{p, \mu \nu}^{J, V \partial, 2}$ & $\mathcal{Q}^{J}$ & $\mathcal{Q}_{\mu}^{J}$ & $\mathcal{Q}_{(\mu \nu)}^{J}$ \\
\hline $\mathfrak{R}$-charge: & $J$ & $J+2$ & $J+4$ & $J+2$ & $J+1$ & $J+3$ & $J$ & $J-1$ & $J-2$ \\
Length $L:$ & $J+2$ & $J+4$ & $J+4$ & $J+2$ & $J+2$ & $J+4$ & $J$ & $J$ & $J$ \\
\hline
\end{tabular}

\section{Two-Point and Three-Point Correlators}

In the following, we will compute two-point and three-point correlators of vacuum operators, operators with zero-momentum excitations, and two-magnon operators, at leading order in the Yang-Mills coupling $g_{\mathrm{YM}}$. The three-point correlators yield the structure constants that will later be needed for the OPE analysis of four-point correlators. Here and in everything that follows, we omit all gauge group factors as well as all factors of $1 /(2 \pi)^{2}$ from propagators $(2.4)$. Keeping this in mind, the classical contractions for the twisted fields are:

$$
\begin{aligned}
& \left\langle\phi\left(x_{1}\right) \phi\left(x_{2}\right)\right\rangle=1, \quad\left\langle V_{\mu}\left(x_{1}\right) V_{\nu}\left(x_{2}\right)\right\rangle=-\frac{\eta_{\mu \nu}}{x_{12}^{2}}, \quad\left\langle B\left(x_{1}\right) B\left(x_{2}\right)\right\rangle=0, \\
& \left\langle\phi\left(x_{1}\right) V_{\mu}\left(x_{2}\right)\right\rangle=-\sqrt{2} \frac{x_{12, \mu}}{x_{12}^{2}}, \quad\left\langle\phi\left(x_{1}\right) B\left(x_{2}\right)\right\rangle=\sqrt{2} \frac{1}{x_{12}^{2}}, \quad\left\langle V_{\mu}\left(x_{1}\right) B\left(x_{2}\right)\right\rangle=0 .
\end{aligned}
$$

The contractions for twisted fields with twisted versions of derivative fields similarly follow by adding the twisting factor $X_{1} \cdot X_{2}$ to the untwisted propagators, for instance

$$
\left\langle\left(X_{1} \cdot \Phi\left(x_{1}\right)\right)\left(X_{2} \cdot D_{\mu} \Phi\left(x_{2}\right)\right)\right\rangle=-2 \frac{x_{12, \mu}}{x_{12}^{4}}\left(X_{1} \cdot X_{2}\right) .
$$

Two-Point Functions. For non-zero momenta, the two-magnon states $(2.18)$ are annihilated by the special conformal generator $\hat{\kappa}_{\mu}(2.15)$. They are thus proper conformal primaries of the twisted conformal algebra. Hence their two-point functions take the standard form as dictated by conformal symmetry: ${ }^{6}$

$$
\left\langle\mathcal{O}_{p, 1}^{J} \mathcal{O}_{q, 2}^{J}\right\rangle=\left\langle\mathcal{O}_{p, 1}^{J, \partial \partial} \mathcal{O}_{q, 2}^{J, \partial \partial}\right\rangle=\frac{\delta_{p, q}}{x_{12}^{4}}
$$

\footnotetext{
${ }^{5}$ For states with identical anomalous dimension, orthogonality may not be preserved by the twisting. In fact, states with scalar zero-momentum excitations $V_{\mu}$ or $B$, which we will consider below, are not orthogonal to the vacuum operators $\mathcal{Q}$, even though they are orthogonal before twisting.

${ }^{6}$ The two-point functions for the operators $\mathcal{O}^{J, V \partial, 1}$ and $\mathcal{O}^{J, V \partial, 2}$ get non-trivial contributions from fermion terms not displayed in $(2.18)$. For $\mathcal{O}_{p}^{J, V \partial, 1}$, reducing to the bosonic terms gives $(1 / 2+(1+\cos (p)) /(J+3))$ times the full two-point function. For $\mathcal{O}^{J, V \partial, 2}$, reducing to the bosonic terms gives $1 / 2$ of the full two-point function.
} 


$$
\begin{gathered}
\left\langle\mathcal{O}_{p,(\mu \nu), 1}^{J, V V} \mathcal{O}_{q,(\rho \sigma), 2}^{J, V V}\right\rangle=\left\langle\mathcal{O}_{p,(\mu \nu), 1}^{J, \partial \partial} \mathcal{O}_{q,(\rho \sigma), 2}^{J, \partial \partial}\right\rangle=\frac{\delta_{p, q}}{x_{12}^{4}} I_{12, \rho(\mu} I_{12, \nu) \sigma} \\
\left\langle\mathcal{O}_{p, \mu \nu, 1}^{J, V \partial, 1} \mathcal{O}_{q, \rho \sigma, 2}^{J, V \partial, 1}\right\rangle=\left\langle\mathcal{O}_{p, \mu \nu, 1}^{J, V \partial, 2} \mathcal{O}_{q, \rho \sigma, 2}^{J, V \partial, 2}\right\rangle=\frac{\delta_{p, q}}{x_{12}^{4}} I_{12, \mu \rho} I_{12, \nu \sigma}
\end{gathered}
$$

Here,

$$
I_{i j, \alpha \beta} \equiv I_{\alpha \beta}\left(x_{i j}\right), \quad I_{\alpha \beta}(x)=\eta_{\alpha \beta}-\frac{2 x_{\alpha} x_{\beta}}{x^{2}}
$$

is the inversion tensor, and parentheses denote traceless symmetrization with unit weight:

$$
x_{(\mu \nu)} \equiv \frac{1}{2} x_{\mu \nu}+\frac{1}{2} x_{\nu \mu}-\frac{1}{4} \eta_{\mu \nu} x_{\rho}^{\rho} .
$$

All other two-point functions among two-magnon states with non-zero momenta vanish.

Two Vacua. By explicit computation, one finds that the three-point functions of two-magnon states (with non-zero momentum) and two vacuum states of the same weight are:

$$
\begin{gathered}
\left\langle\mathcal{O}_{p, 1}^{J} \mathcal{Q}_{2}^{k} \mathcal{Q}_{3}^{k}\right\rangle=\left\langle\mathcal{O}_{p, 1}^{J, \partial \partial} \mathcal{Q}_{2}^{k} \mathcal{Q}_{3}^{k}\right\rangle=\mathcal{C}_{p}^{J} k^{2} \frac{x_{23}^{2}}{x_{12}^{2} x_{13}^{2}} \\
\left\langle\mathcal{O}_{p,(\mu \nu), 1}^{J, V V} \mathcal{Q}_{2}^{k} \mathcal{Q}_{3}^{k}\right\rangle=\left\langle\mathcal{O}_{p,(\mu \nu), 1}^{J, \partial \partial} \mathcal{Q}_{2}^{k} \mathcal{Q}_{3}^{k}\right\rangle=\mathcal{C}_{p}^{J} 2 k^{2} Y_{(\mu, 12,13} Y_{\nu, 12,13} \\
\left\langle\mathcal{O}_{p, \mu \nu, 1}^{J, V \partial, 1} \mathcal{Q}_{2}^{k} \mathcal{Q}_{3}^{k}\right\rangle=\left\langle\mathcal{O}_{p, \mu \nu, 1}^{J, V \partial, 2} \mathcal{Q}_{2}^{k} \mathcal{Q}_{3}^{k}\right\rangle=\mathcal{C}_{p}^{J} 2 i k^{2} Y_{\mu, 12,13} Y_{\nu, 12,13}
\end{gathered}
$$

with

$$
Y_{\alpha}(x, y)=\frac{x^{\alpha}}{x^{2}}-\frac{y^{\alpha}}{y^{2}}, \quad Y_{\alpha, i j, k l}=Y_{\alpha}\left(x_{i j}, x_{k l}\right) .
$$

In this case, all three operators are proper conformal primaries (they are annihilated by $\hat{\kappa}_{\mu}$ ), and hence these correlation functions are of the standard form as dictated by conformal symmetry. Here, the coefficient depending on the charge and momentum is

$$
\mathcal{C}_{p}^{J}=\frac{1}{\sqrt{J+3}} \frac{-e^{-i p / 2}}{\left(1+e^{i(J+2) p / 2}\right)^{2}} .
$$

The two-magnon states $(2.18)$ are all mutually orthogonal as well as orthogonal to the vacuum states and all their descendants. Hence, the coefficients of the two-magnon states in the OPE of two vacuum operators can simply be extracted from the three- and two-point functions,

$$
\left\langle\mathcal{O}_{1}\left(x_{1}\right) \mathcal{O}_{2}\left(x_{2}\right) \mathcal{O}_{3}\left(x_{3}\right)\right\rangle \stackrel{x_{2} \rightarrow x_{3}}{\longrightarrow} C_{23}^{1}\left(x_{23}\right)\left\langle\mathcal{O}_{1}\left(x_{1}\right) \mathcal{O}_{1}\left(x_{2}\right)\right\rangle+\ldots
$$

where the ellipsis stands for higher-order terms in $x_{23}$ that stem from descendants of $\mathcal{O}_{1}$. The resulting OPE coefficients for two vacuum operators read

$$
\begin{gathered}
C_{k ; k}^{J, p}=C_{k ; k}^{J, p, \partial \partial}=\mathcal{C}_{p}^{J} k^{2} x_{12}^{2}, \\
C_{k ; k}^{J, p, V V,(\mu \nu)}=C_{k ; k}^{J, p, \partial \partial,(\mu \nu)}=\mathcal{C}_{p}^{J} 2 k^{2} x_{12}^{(\mu} x_{12}^{\nu)}, \\
C_{k ; k}^{J, p, V \partial, 1, \mu \nu}=C_{k ; k}^{J, p, V \partial, 2, \mu \nu}=\mathcal{C}_{p}^{J} 2 i k^{2} x_{12}^{\mu} x_{12}^{\nu} .
\end{gathered}
$$


One Excitation. Zero-momentum one-excitation operators transform non-trivially under $\hat{\kappa}_{\mu}$, hence they are not proper conformal primaries, and their correlation functions will not be of the familiar form as dictated by conformal symmetry. By direct computation, one finds the following three-point functions of two-magnon states (with non-zero momentum), a one-excitation BPS state $\mathcal{Q}_{\rho}^{k}$ and a vacuum state $\mathcal{Q}^{k}$ :

$$
\begin{aligned}
\left\langle\mathcal{O}_{p, 1}^{J} \mathcal{Q}_{\rho, 2}^{k} \mathcal{Q}_{3}^{k}\right\rangle & =\mathcal{C}_{p}^{J} \sqrt{2} k \frac{x_{23}^{2}}{x_{12}^{2} x_{13}^{2}}\left(\frac{1}{2} \boldsymbol{Y}_{\rho, 21,23}-Y_{\rho, 21,23}\right) \\
\left\langle\mathcal{O}_{p,(\mu \nu), 1}^{J, V V} \mathcal{Q}_{\rho, 2}^{k} \mathcal{Q}_{3}^{k}\right\rangle & =\mathcal{C}_{p}^{J} \sqrt{2} k\left(\boldsymbol{Y}_{\rho, 21,23} Y_{(\mu, 12,13} Y_{\nu), 12,13}-\frac{2}{x_{12}^{2}} I_{12, \rho(\mu} Y_{\nu), 12,13}\right) \\
\left\langle\mathcal{O}_{p, 1}^{J, \partial \partial} \mathcal{Q}_{\rho, 2}^{k} \mathcal{Q}_{3}^{k}\right\rangle & =\mathcal{C}_{p}^{J} \sqrt{2} k \frac{x_{23}^{2}}{x_{12}^{2} x_{13}^{2} \frac{1}{2} \boldsymbol{Y}_{\rho, 21,23}} \\
\left\langle\mathcal{O}_{p,(\mu \nu), 1}^{J, \partial \partial} \mathcal{Q}_{\rho, 2}^{k} \mathcal{Q}_{3}^{k}\right\rangle & =\mathcal{C}_{p}^{J} \sqrt{2} k \boldsymbol{Y}_{\rho, 21,23} Y_{(\mu, 12,13} Y_{\nu), 12,13}, \\
\left\langle\mathcal{O}_{p, \mu \nu, 1}^{J, V \partial, 1} \mathcal{Q}_{\rho, 2}^{k} \mathcal{Q}_{3}^{k}\right\rangle & =\mathcal{C}_{p}^{J} \sqrt{2} i k\left(\boldsymbol{Y}_{\rho, 21,23} Y_{\mu, 12,13} Y_{\nu, 12,13}-\frac{1}{x_{12}^{2}} I_{12, \rho \mu} Y_{\nu, 12,13}\right) \\
\left\langle\mathcal{O}_{p, \mu \nu, 1}^{J, V \partial, 2} \mathcal{Q}_{\rho, 2}^{k} \mathcal{Q}_{3}^{k}\right\rangle & =\mathcal{C}_{p}^{J} \sqrt{2 i} k\left(\boldsymbol{Y}_{\rho, 21,23} Y_{\mu, 12,13} Y_{\nu, 12,13}-\frac{1}{x_{12}^{2}} I_{12, \rho \mu} Y_{\nu, 12,13}\right)
\end{aligned}
$$

Here,

$$
\boldsymbol{Y}_{\rho, i j, i k}=\left(L Y_{\rho, i j, i k}+2 k \frac{x_{i k}^{\rho}}{x_{i k}^{2}}\right)
$$

The resulting OPE coefficients are

$$
\begin{aligned}
C_{k, \rho ; k}^{J, p} & =\mathcal{C}_{p}^{J} \sqrt{2} k(2 k-L+2) \frac{1}{2} x_{12, \rho}, \\
C_{k, \rho ; k}^{J, p, V V,(\mu \nu)} & =\mathcal{C}_{p}^{J} \sqrt{2} k\left(\frac{(2 k-L) x_{12}^{(\mu} x_{12}^{\nu)} x_{12, \rho}}{x_{12}^{2}}+2 x_{12}^{(\mu} \eta_{\rho}^{\nu)}\right), \\
C_{k, \rho ; k}^{J, p, \partial \partial} & =\mathcal{C}_{p}^{J} \sqrt{2} k(2 k-L) \frac{1}{2} x_{12, \rho}, \\
C_{k, \rho ; k}^{J, p, \partial \partial,(\mu \nu)} & =\mathcal{C}_{p}^{J} \sqrt{2} k(2 k-L) \frac{x_{12}^{(\mu} x_{12}^{\nu)} x_{12, \rho}}{x_{12}^{2}} \\
C_{k, \rho ; k}^{J, p, V \partial, 1, \mu \nu} & =\mathcal{C}_{p}^{J} \sqrt{2} i k x_{12}^{\nu}\left(\frac{(2 k-L) x_{12}^{\mu} x_{12, \rho}}{x_{12}^{2}}+\eta_{\rho}^{\mu}\right) \\
C_{k, \rho ; k}^{J, p, V \partial, 2, \mu \nu} & =\mathcal{C}_{p}^{J} \sqrt{2} i k x_{12}^{\nu}\left(\frac{(2 k-L) x_{12}^{\mu} x_{12, \rho}}{x_{12}^{2}}+\eta_{\rho}^{\mu}\right) .
\end{aligned}
$$

Two Excitations. The three-point functions of two-magnon states (with non-zero momentum) and two one-excitation BPS states read:

$$
\begin{aligned}
&\left\langle\mathcal{O}_{p, 1}^{J} \mathcal{Q}_{\rho, 2}^{k} \mathcal{Q}_{\sigma, 3}^{k}\right\rangle= \mathcal{C}_{p}^{J} \frac{x_{23}^{2}}{x_{12}^{2} x_{13}^{2}}\left[\left(\frac{1}{2} \boldsymbol{Y}_{\rho, 21,23} \boldsymbol{Y}_{\sigma, 31,32}-\frac{2 k-L}{2 x_{23}^{2}} I_{23, \rho \sigma}\right)\right. \\
&\left.\quad-\boldsymbol{Y}_{\rho, 21,23} Y_{\sigma, 31,32}-Y_{\rho, 21,23} \boldsymbol{Y}_{\sigma, 31,32}-\frac{I_{12, \rho \mu} I_{13}{ }_{\sigma} \sigma}{x_{23}^{2}}\right], \\
&\left\langle\mathcal{O}_{p,(\mu \nu), 1}^{J, V V} \mathcal{Q}_{\rho, 2}^{k} \mathcal{Q}_{\sigma, 3}^{k}\right\rangle= \mathcal{C}_{p}^{J}\left[\left(\boldsymbol{Y}_{\rho, 21,23} \boldsymbol{Y}_{\sigma, 31,32}-\frac{2 k-L}{x_{23}^{2}} I_{23, \rho \sigma}\right) Y_{(\mu, 12,13} Y_{\nu), 12,13}\right. \\
&\left.-\boldsymbol{Y}_{\rho, 21,23} \frac{2}{x_{13}^{2}} I_{13, \sigma(\mu} Y_{\nu), 13,12}-\frac{2}{x_{12}^{2}} I_{12, \rho(\mu} Y_{\nu), 12,13} \boldsymbol{Y}_{\sigma, 31,32}-\frac{2 I_{12, \rho(\mu} I_{13, \nu) \sigma}}{x_{12}^{2} x_{13}^{2}}\right],
\end{aligned}
$$




$$
\begin{aligned}
& \left\langle\mathcal{O}_{p, 1}^{J, \partial \partial} \mathcal{Q}_{\rho, 2}^{k} \mathcal{Q}_{\sigma, 3}^{k}\right\rangle=\mathcal{C}_{p}^{J} \frac{x_{23}^{2}}{x_{12}^{2} x_{13}^{2}}\left(\frac{1}{2} \boldsymbol{Y}_{\rho, 21,23} \boldsymbol{Y}_{\sigma, 31,32}-\frac{2 k-L}{2 x_{23}^{2}} I_{23, \rho \sigma}\right), \\
& \left\langle\mathcal{O}_{p,(\mu \nu), 1}^{J, \partial \partial} \mathcal{Q}_{\rho, 2}^{k} \mathcal{Q}_{\sigma, 3}^{k}\right\rangle=\mathcal{C}_{p}^{J}\left(\boldsymbol{Y}_{\rho, 21,23} \boldsymbol{Y}_{\sigma, 31,32}-\frac{2 k-L}{x_{23}^{2}} I_{23, \rho \sigma}\right) Y_{(\mu, 12,13} Y_{\nu), 12,13} \\
& \left\langle\mathcal{O}_{p, \mu \nu, 1}^{J, V \partial, 1} \mathcal{Q}_{\rho, 2}^{k} \mathcal{Q}_{\sigma, 3}^{k}\right\rangle=\mathcal{C}_{p}^{J} i\left[\left(\boldsymbol{Y}_{\rho, 21,23} \boldsymbol{Y}_{\sigma, 31,32}-\frac{2 k-L}{x_{23}^{2}} I_{23, \rho \sigma}\right) Y_{\mu, 12,13} Y_{\nu, 12,13}\right. \\
& \left.-\boldsymbol{Y}_{\rho, 21,23} \frac{1}{x_{13}^{2}} I_{13, \sigma \mu} Y_{\nu, 13,12}-\frac{1}{x_{12}^{2}} I_{12, \rho \mu} Y_{\nu, 12,13} \boldsymbol{Y}_{\sigma, 31,32}\right], \\
& \left\langle\mathcal{O}_{p, \mu \nu, 1}^{J, V \partial, 2} \mathcal{Q}_{\rho, 2}^{k} \mathcal{Q}_{\sigma, 3}^{k}\right\rangle=\mathcal{C}_{p}^{J} i\left[\left(\boldsymbol{Y}_{\rho, 21,23} \boldsymbol{Y}_{\sigma, 31,32}-\frac{2 k-L}{x_{23}^{2}} I_{23, \rho \sigma}\right) Y_{\mu, 12,13} Y_{\nu, 12,13}\right. \\
& \left.-\boldsymbol{Y}_{\rho, 21,23} \frac{1}{x_{13}^{2}} I_{13, \sigma \mu} Y_{\nu, 13,12}-\frac{1}{x_{12}^{2}} I_{12, \rho \mu} Y_{\nu, 12,13} \boldsymbol{Y}_{\sigma, 31,32}\right] .
\end{aligned}
$$

The resulting OPE coefficients are

$$
\begin{aligned}
C_{k, \rho ; k, \sigma}^{J, p} & =-\mathcal{C}_{p}^{J} \frac{(2 k-L+2)}{2}\left(\boldsymbol{I}_{\rho \sigma, 12}+\frac{2 x_{12, \rho} x_{12, \sigma}}{x_{12}^{2}}\right) \\
C_{k, \rho ; k, \sigma}^{J, p, V V,(\mu \nu)} & =-\mathcal{C}_{p}^{J}\left((2 k-L) \frac{x_{12}^{(\mu}}{x_{12}^{2}}\left(2\left(x_{12, \sigma} \eta_{\rho}^{\nu)}+x_{12, \rho} \eta_{\sigma}^{\nu)}\right)+x_{12}^{\nu)} \boldsymbol{I}_{\rho \sigma, 12}\right)+2 \eta_{\rho}^{(\mu} \eta_{\sigma}^{\nu)}\right), \\
C_{k, \rho ; k, \sigma}^{J, p, \partial \partial} & =-\mathcal{C}_{p}^{J} \frac{(2 k-L)}{2} \boldsymbol{I}_{\rho \sigma, 12} \\
C_{k, \rho ; k, \sigma}^{J, p, \partial \partial,(\mu \nu)} & =-\mathcal{C}_{p}^{J}(2 k-L) \frac{x_{12}^{(\mu} x_{12}^{\nu)}}{x_{12}^{2}} \boldsymbol{I}_{\rho \sigma, 12}, \\
C_{k, \rho ; k, \sigma}^{J, p, V \partial, 1, \mu \nu} & =-\mathcal{C}_{p}^{J} i(2 k-L) \frac{x_{12}^{\nu}}{x_{12}^{2}}\left(\left(x_{12, \sigma} \eta_{\rho}^{\mu}+x_{12, \rho} \eta_{\sigma}^{\mu}\right)+x_{12}^{\mu} \boldsymbol{I}_{\rho \sigma, 12}\right), \\
C_{k, \rho ; k, \sigma}^{J, p, V \partial, 2, \mu \nu} & =-\mathcal{C}_{p}^{J} i(2 k-L) \frac{x_{12}^{\nu}}{x_{12}^{2}}\left(\left(x_{12, \sigma} \eta_{\rho}^{\mu}+x_{12, \rho} \eta_{\sigma}^{\mu}\right)+x_{12}^{\mu} \boldsymbol{I}_{\rho \sigma, 12}\right),
\end{aligned}
$$

where

$$
\boldsymbol{I}^{\rho \sigma}(x) \equiv I^{\rho \sigma}(x)+\frac{(2 k-L) x^{\rho} x^{\sigma}}{x^{2}} .
$$

Even though this will not be needed in the subsequent analysis, we note that for two zeromomentum excitations on one of the two external half-BPS states, the OPE coefficient of the two-magnon operator $\mathcal{O}_{p}^{J}$ reads

$$
C_{k,(\rho \sigma) ; k}^{J, p}=\mathcal{C}_{p}^{J} k(2 k-L+2)(2 k-L) \frac{x_{12,(\rho} x_{12, \sigma)}}{x_{12}^{2}} .
$$

The Coefficient Function. Using the on-shell condition $(2.19)$, the OPE coefficent function $(3.8)$ can be rewritten as

$$
\mathcal{C}_{p}^{J}=\frac{-1}{2 \sqrt{J+3}\left(\cos (p / 2)+e^{i p(J+3) / 2}\right)} .
$$

It has a few interesting properties. First of all, because $p$ is a multiple of $2 \pi /(J+3)$, the term $e^{i p(J+3) / 2}$ alternates between -1 and +1 when $p$ is varied. The value +1 is assumed when $p$ is a multiple of $4 \pi /(J+3)$. For fixed $J$, the coefficient $\mathcal{C}_{p}^{J}$ therefore has two branches 


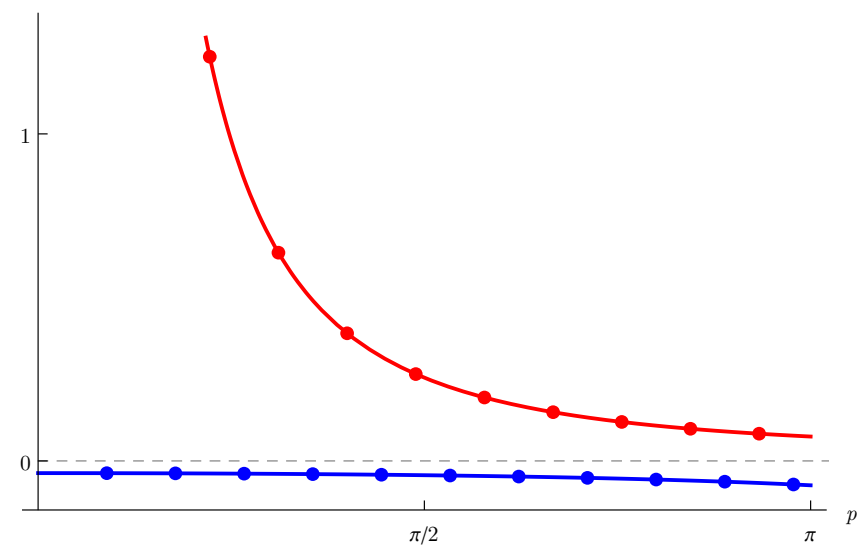

Figure 1: The two branches of the OPE coefficient function $\mathcal{C}_{p}^{J}$, here displayed for $J=42$. On the lower branch, $p$ is a multiple of $4 \pi /(J+3)$, and therefore $\mathcal{C}_{p}^{J} \sim(J+3)^{-1 / 2}$. On the upper branch, $\mathcal{C}_{p}^{J}$ becomes large for small $p$, due to its small denominator. For the smallest two values $p=2 \pi\{1,3\} /(J+3)$ on the upper branch, the coefficient is off the chart at $\mathcal{C}_{p}^{J} \approx\{30.6,3.41\}$.

(see Figure 1). Since $p$ takes values between 0 and $\pi$, the coefficient is small on the "+" branch, $\mathcal{C}_{p,+}^{J} \approx-1 / 4 \sqrt{J+3}$. On the "-" branch, $\mathcal{C}_{p}^{J}$ becomes large when $p$ is small, $\mathcal{C}_{p,-}^{J} \approx 4 / \sqrt{J+3} p^{2}$. In fact, for large values of $J$, the smallest momentum $p=2 \pi /(J+3)$ is strongly dominating (see Figure 2). The reason for the alternating behavior of $\mathcal{C}_{p}^{J}$ is that the coefficient of the "local" two-excitation operator

$$
\operatorname{tr}\left[V_{\mu}, \phi, \ldots, \phi, V_{\nu}, \phi, \ldots, \phi\right]
$$

in the OPE of two vacuum (or zero-momentum) operators has a part that is independent of the distance between the two excitations, and a part that is proportional to that distance. In the symmetric traceless two-magnon operator (2.18), the coefficients of these local operators (i) sum to zero, and (ii) depending on the momentum $p$, alternate between (almost) odd and even functions of the distance between the two excitations. Hence, the distance-independent part of the OPE coefficient drops out, while the linear part is alternatingly enhanced/suppressed. The same applies to the other types of two-magnon states.

\section{Four-Point Correlators}

The one-loop correlator of four chiral primary operators $\mathcal{P}_{i}=\operatorname{tr}\left[\left(X_{i} \cdot \Phi\right)^{k}\right]\left(x_{i}\right)$ of weight $k$, for general orientations $X_{i}$ with $X_{i}^{2}=0$, is given by $[16,9$

$$
\left\langle\mathcal{P}_{1} \mathcal{P}_{2} \mathcal{P}_{3} \mathcal{P}_{4}\right\rangle^{\text {one-loop }}=\frac{\lambda}{8 \pi^{2}} I^{(1)}(s, t) k^{4} \mathcal{R} \sum_{\substack{j, \ell, m \geq 0 \\ j+\ell+m=k-2}} \mathcal{X}^{j} \mathcal{Y}^{\ell} \mathcal{Z}^{m}
$$

where

$$
\mathcal{X}=\frac{X_{1} \cdot X_{2} X_{3} \cdot X_{4}}{x_{12}^{2} x_{34}^{2}}, \quad \mathcal{Y}=\frac{X_{1} \cdot X_{3} X_{2} \cdot X_{4}}{x_{13}^{2} x_{24}^{2}}, \quad \mathcal{Z}=\frac{X_{1} \cdot X_{4} X_{2} \cdot X_{3}}{x_{14}^{2} x_{23}^{2}}
$$

are the three possible combinations of propagator structures for four-points,

$$
\mathcal{R}=s \mathcal{X}^{2}+\mathcal{Y}^{2}+t \mathcal{Z}^{2}+(s-t-1) \mathcal{Y} \mathcal{Z}+(1-s-t) \mathcal{X} \mathcal{Z}+(t-s-1) \mathcal{X} \mathcal{Y}
$$

is a universal prefactor due to supersymmetry,

$$
s=\frac{x_{12}^{2} x_{34}^{2}}{x_{13}^{2} x_{24}^{2}}, \quad t=\frac{x_{14}^{2} x_{23}^{2}}{x_{13}^{2} x_{24}^{2}}
$$



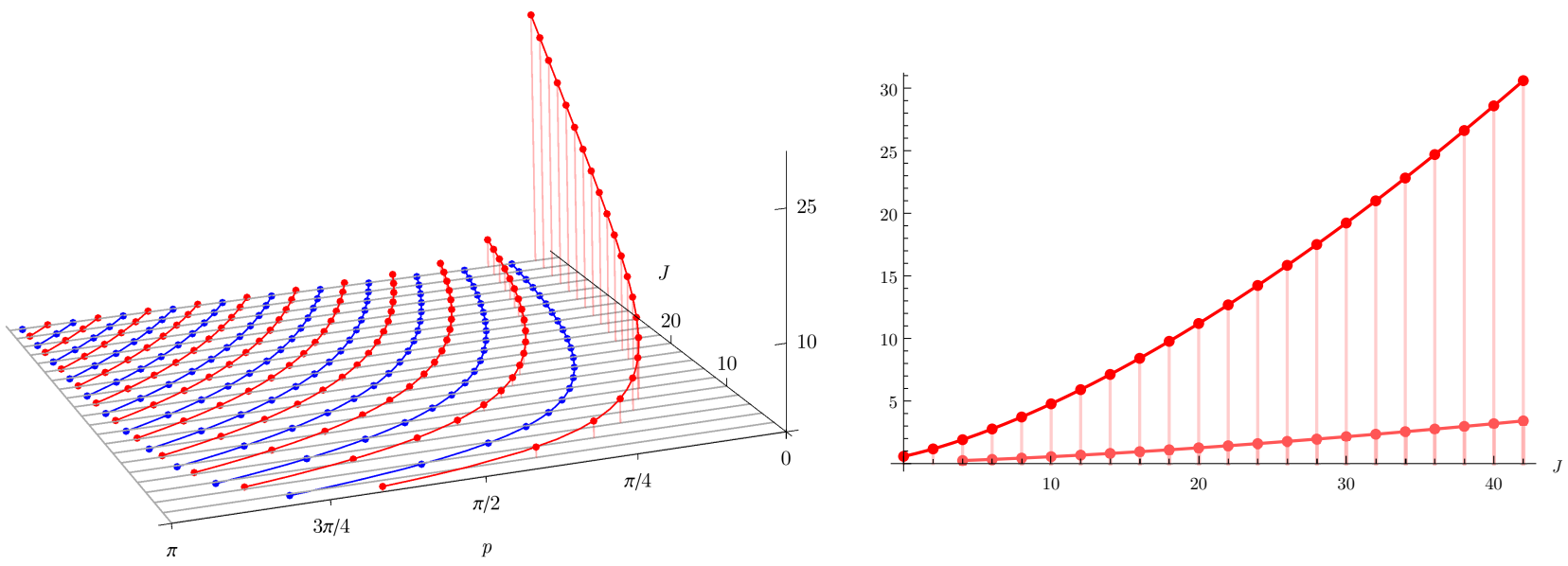

Figure 2: The coefficient function $\mathcal{C}_{p}^{J}$. The plot on the left runs over all admissible momenta $p$ for $J \leq 42$. Every dot stands for one two-magnon supermultiplet. The "-" branch is plotted in red, the "+" branch is plotted in blue. For each $J$, the smallest momentum $p=2 \pi /(J+3)$ dominates. The plot on the right shows the two largest values of the coefficient $\mathcal{C}_{p}^{J}$ on the dominant " -" branch $(p=2 \pi /(J+3)$ and $p=6 \pi /(J+3))$, for $J \leq 42$.

are the conformally invariant cross-ratios, and

$$
I^{(1)}(s, t)=\frac{x_{13}^{2} x_{24}^{2}}{2 \pi^{2}} \int \frac{d^{4} x_{5}}{x_{15}^{2} x_{25}^{2} x_{35}^{2} x_{45}^{2}}
$$

is the conformal one-loop scalar box integral, which has been computed in [17] and is most conveniently written as 18

$$
I^{(1)}(s, t)=-\frac{1}{z-\bar{z}}\left(2\left(\operatorname{Li}_{2}(z)-\operatorname{Li}_{2}(\bar{z})\right)-\left(\operatorname{Li}_{1}(z)-\operatorname{Li}_{1}(\bar{z})\right) \log s\right)
$$

where the cross ratios are parametrized in the standard way

$$
s=z \bar{z}, \quad t=(1+z)(1+\bar{z}) .
$$

We consider Euclidean signature, which means that $z$ and $\bar{z}$ are mutually complex conjugate.

\subsection{Twisted Correlators}

The vacuum operators $\mathcal{Q}$ are deliberately oriented such that $X_{i} \cdot X_{j}=X^{\phi} \cdot X^{\phi}=x_{i j}^{2}$, which sets $\mathcal{X}=\mathcal{Y}=\mathcal{Z}=1$, and therefore $\mathcal{R}=0$, in accordance with the fact that correlation functions of vacuum operators are protected:

$$
\left\langle\mathcal{Q}_{1} \mathcal{Q}_{2} \mathcal{Q}_{3} \mathcal{Q}_{4}\right\rangle^{\text {one-loop }}=0
$$

Adding zero-momentum excitations $V_{\mu}$ on top of the vacuum $\phi$ can be achieved by setting $X_{i}=X^{\phi}+\varepsilon_{i}^{\mu} X^{V_{\mu}}$. To leading order in $\varepsilon_{i}$, the condition $X_{i} \cdot X_{i}=0$ is still satisfied, and the four-point correlator expands to

$$
\begin{aligned}
&\left\langle\mathcal{P}_{1} \mathcal{P}_{2} \mathcal{P}_{3} \mathcal{P}_{4}\right\rangle=\left\langle\mathcal{Q}_{1} \mathcal{Q}_{2} \mathcal{Q}_{3} \mathcal{Q}_{4}\right\rangle+\varepsilon_{1}^{\mu} k\left\langle\mathcal{Q}_{\mu, 1} \mathcal{Q}_{2} \mathcal{Q}_{3} \mathcal{Q}_{4}\right\rangle+\cdots+\varepsilon_{1}^{\mu} \varepsilon_{2}^{\nu} k^{2}\left\langle\mathcal{Q}_{\mu, 1} \mathcal{Q}_{\nu, 2} \mathcal{Q}_{3} \mathcal{Q}_{4}\right\rangle \\
&+\cdots+\varepsilon_{1}^{\mu} \varepsilon_{2}^{\nu} \varepsilon_{3}^{\rho} k^{3}\left\langle\mathcal{Q}_{\mu, 1} \mathcal{Q}_{\nu, 2} \mathcal{Q}_{\rho, 3} \mathcal{Q}_{4}\right\rangle+\cdots+\varepsilon_{1}^{\mu} \varepsilon_{2}^{\nu} \varepsilon_{3}^{\rho} \varepsilon_{4}^{\sigma} k^{4}\left\langle\mathcal{Q}_{\mu, 1} \mathcal{Q}_{\nu, 2} \mathcal{Q}_{\rho, 3} \mathcal{Q}_{\sigma, 4}\right\rangle+\ldots
\end{aligned}
$$


The dependence on $\varepsilon_{i}^{\mu}$ enters the correlator (4.1) only through the propagator structures $\mathcal{X}, \mathcal{Y}$, and $\mathcal{Z}$, which expand to

$$
\begin{aligned}
& \mathcal{X}=\left(1+\frac{\sqrt{2}\left(\varepsilon_{1}-\varepsilon_{2}\right) \cdot x_{12}-\varepsilon_{1} \cdot \varepsilon_{2}}{x_{12}^{2}}\right)\left(1+\frac{\sqrt{2}\left(\varepsilon_{3}-\varepsilon_{4}\right) \cdot x_{34}-\varepsilon_{3} \cdot \varepsilon_{4}}{x_{34}^{2}}\right), \\
& \mathcal{Y}=\left(1+\frac{\sqrt{2}\left(\varepsilon_{1}-\varepsilon_{3}\right) \cdot x_{13}-\varepsilon_{1} \cdot \varepsilon_{3}}{x_{13}^{2}}\right)\left(1+\frac{\sqrt{2}\left(\varepsilon_{2}-\varepsilon_{4}\right) \cdot x_{24}-\varepsilon_{2} \cdot \varepsilon_{4}}{x_{24}^{2}}\right), \\
& \mathcal{Z}=\left(1+\frac{\sqrt{2}\left(\varepsilon_{2}-\varepsilon_{3}\right) \cdot x_{23}-\varepsilon_{2} \cdot \varepsilon_{3}}{x_{23}^{2}}\right)\left(1+\frac{\sqrt{2}\left(\varepsilon_{1}-\varepsilon_{4}\right) \cdot x_{14}-\varepsilon_{1} \cdot \varepsilon_{4}}{x_{14}^{2}}\right) .
\end{aligned}
$$

We will denote the expansion coefficients of the universal prefactor $\mathcal{R}$ by $\mathcal{R}^{i, j, \ldots}$ :

$$
\mathcal{R}=\sum_{i, j=1}^{4} \varepsilon_{i} \varepsilon_{j} \mathcal{R}^{i, j}+\sum_{i, j, k=1}^{4} \varepsilon_{i} \varepsilon_{j} \varepsilon_{k} \mathcal{R}^{i, j, k}+\varepsilon_{1} \varepsilon_{2} \varepsilon_{3} \varepsilon_{4} \mathcal{R}^{1,2,3,4}+\ldots
$$

The expansion only starts at quadratic order in the parameters $\varepsilon_{i}$, hence also correlators with a single excitation are protected:

$$
\left\langle\mathcal{Q}_{\mu, 1} \mathcal{Q}_{2} \mathcal{Q}_{3} \mathcal{Q}_{4}\right\rangle^{\text {one-loop }}=0
$$

At quadratic order, all dependence on the parameters $\varepsilon_{i}$ is absorbed by the prefactor $\mathcal{R}$. Therefore, the sum over $\mathcal{X}^{j} \mathcal{Y}^{\ell} \mathcal{Z}^{m}$ trivially evaluates to $k(k-1) / 2$, and one finds for the correlators with two excitations:

$$
\begin{aligned}
& \left\langle\mathcal{Q}_{\mu, 1} \mathcal{Q}_{\nu, 2} \mathcal{Q}_{3} \mathcal{Q}_{4}\right\rangle^{\text {one-loop }}=\frac{\lambda}{8 \pi^{2}} I^{(1)}(s, t) \frac{k^{3}(k-1)}{2} \mathcal{R}_{\mu \nu}^{1,2}, \\
& \left\langle\mathcal{Q}_{\mu, 1} \mathcal{Q}_{2} \mathcal{Q}_{3} \mathcal{Q}_{\sigma, 4}\right\rangle^{\text {one-loop }}=\frac{\lambda}{8 \pi^{2}} I^{(1)}(s, t) \frac{k^{3}(k-1)}{2} \mathcal{R}_{\mu \sigma}^{1,4}, \quad \text { etc. },
\end{aligned}
$$

where

$$
\begin{aligned}
\mathcal{R}^{1,2, \mu \nu}=4 \frac{x_{13}^{\mu} x_{24}^{\nu}}{x_{13}^{2} x_{24}^{2}}-4 s \frac{x_{12}^{\mu} x_{12}^{\nu}}{x_{12}^{4}}+4 t \frac{x_{14}^{\mu} x_{23}^{\nu}}{x_{14}^{2} x_{23}^{2}}+2(-1+s+t)\left(\frac{x_{12}^{\nu} x_{14}^{\mu}}{x_{12}^{2} x_{14}^{2}}-\frac{x_{12}^{\mu} x_{23}^{\nu}}{x_{12}^{2} x_{23}^{2}}\right) \\
+2(1+s-t)\left(\frac{x_{12}^{\nu} x_{13}^{\mu}}{x_{12}^{2} x_{13}^{2}}-\frac{x_{12}^{\mu} x_{24}^{\nu}}{x_{12}^{2} x_{24}^{2}}\right)+2(-1+s-t)\left(\frac{x_{13}^{\mu} x_{23}^{\nu}}{x_{13}^{2} x_{23}^{2}}+\frac{x_{14}^{\mu} x_{24}^{\nu}}{x_{14}^{2} x_{24}^{2}}\right),
\end{aligned}
$$

and all other $\mathcal{R}^{i, j}$ are given by similar expressions (see Appendix A).

The correlators $\left\langle\mathcal{Q}_{\mu, 1} \mathcal{Q}_{\nu, 2} \mathcal{Q}_{3} \mathcal{Q}_{\sigma, 4}\right\rangle$ and $\left\langle\mathcal{Q}_{\mu, 1} \mathcal{Q}_{\nu, 2} \mathcal{Q}_{\rho, 3} \mathcal{Q}_{\sigma, 4}\right\rangle$ with three or four excitations are a bit more complicated to obtain than the previous examples. Upon inserting (4.10) into the general formula (4.1), we need to extract the coefficients of cubic and quartic monomials in the parameters $\varepsilon_{i}$. Since the universal prefactor $\mathcal{R}$ starts at quadratic order, we also need to expand the sum over propagator structures to quadratic order. Below, we will consider the double-coincidence limit $\left|x_{12}\right|,\left|x_{34}\right| \ll\left|x_{23}\right|$. In that limit, the expansion of the sum of propagator structures reads:

$$
\begin{aligned}
& \sum_{\substack{j, \ell, m \geq 0 \\
j+\ell+m=k-2}} \mathcal{X}^{j} \mathcal{Y}^{\ell} \mathcal{Z}^{m}=\frac{k(k-1)}{2}+\frac{k(k-1)(k-2)}{6}\left[\sqrt{2}\left(\frac{\left(\varepsilon_{1}-\varepsilon_{2}\right) \cdot x_{12}}{x_{12}^{2}}+\frac{\left(\varepsilon_{3}-\varepsilon_{4}\right) \cdot x_{34}}{x_{34}^{2}}\right)\right. \\
& \left.\quad+\frac{k-1}{2}\left(\frac{\left(\varepsilon_{1}-\varepsilon_{2}\right) \cdot x_{12}}{x_{12}^{2}}+\frac{\left(\varepsilon_{3}-\varepsilon_{4}\right) \cdot x_{34}}{x_{34}^{2}}\right)^{2}-\frac{\varepsilon_{1} \cdot I\left(x_{12}\right) \cdot \varepsilon_{2}}{x_{12}^{2}}-\frac{\varepsilon_{3} \cdot I\left(x_{34}\right) \cdot \varepsilon_{4}}{x_{34}^{2}}\right]
\end{aligned}
$$




$$
+\mathcal{O}\left(\varepsilon_{i}^{2}\right)+\mathcal{O}\left(\varepsilon_{i} \varepsilon_{j} \varepsilon_{k}\right) .
$$

Combining the relevant terms with the coefficients of the prefactor $\mathcal{R}$, we find for the one-loop correlator with three excitations, in the double-coincidence limit:

$$
\begin{aligned}
\left\langle\mathcal{Q}_{\mu, 1} \mathcal{Q}_{\nu, 2} \mathcal{Q}_{3} \mathcal{Q}_{\sigma, 4}\right\rangle^{\text {one-loop }} & =\frac{\lambda}{8 \pi^{2}} I^{(1)}(s, t) \frac{k^{2}(k-1)}{2} . \\
& \cdot\left(\mathcal{R}_{\mu \nu \sigma}^{1,2,4}+\frac{\sqrt{2}(k-2)}{3}\left(\frac{x_{12, \mu}}{x_{12}^{2}} \mathcal{R}_{\nu \sigma}^{2,4}-\frac{x_{12, \nu}}{x_{12}^{2}} \mathcal{R}_{\mu \sigma}^{1,4}+\frac{x_{34, \sigma}}{x_{34}^{2}} \mathcal{R}_{\mu \nu}^{1,2}\right)\right),
\end{aligned}
$$

and for the one-loop correlator with four excitations:

$$
\begin{aligned}
& \left\langle\mathcal{Q}_{\mu, 1} \mathcal{Q}_{\nu, 2} \mathcal{Q}_{\rho, 3} \mathcal{Q}_{\sigma, 4}\right\rangle^{\text {one-loop }}=\frac{\lambda}{8 \pi^{2}} I^{(1)}(s, t) \frac{k(k-1)}{2} . \\
& \cdot\left(\mathcal{R}_{\mu \nu \rho \sigma}^{1,2,3,4}+\frac{(k-2)}{3}\left[\sqrt{2}\left(\frac{x_{12, \mu}}{x_{12}^{2}} \mathcal{R}_{\nu \rho \sigma}^{2,3,4}-\frac{x_{12, \nu}}{x_{12}^{2}} \mathcal{R}_{\mu \rho \sigma}^{1,3,4}+\frac{x_{34, \sigma}}{x_{34}^{2}} \mathcal{R}_{\mu \nu \rho}^{1,2,3}\right)\right.\right. \\
& +(k-1)\left(-\frac{x_{12, \mu} x_{12, \nu}}{x_{12}^{4}} \mathcal{R}_{\rho \sigma}^{3,4}+\frac{x_{12, \mu} x_{34, \rho}}{x_{12}^{2} x_{34}^{2}} \mathcal{R}_{\nu \sigma}^{2,4}-\frac{x_{12, \mu} x_{34, \sigma}}{x_{12}^{2} x_{34}^{2}} \mathcal{R}_{\nu \rho}^{2,3}-\frac{x_{12, \nu} x_{34, \rho}}{x_{12}^{2} x_{34}^{2}} \mathcal{R}_{\mu \sigma}^{1,4}\right. \\
& \left.\left.\left.\quad+\frac{x_{12, \nu} x_{34, \sigma}}{x_{12}^{2} x_{34}^{2}} \mathcal{R}_{\mu \rho}^{1,3}-\frac{x_{34, \rho} x_{34, \sigma}}{x_{12}^{4}} \mathcal{R}_{\mu \nu}^{1,2}\right)-\frac{I_{12, \mu \nu}}{x_{12}^{2}} \mathcal{R}_{\rho \sigma}^{3,4}-\frac{I_{34, \rho \sigma}}{x_{34}^{2}} \mathcal{R}_{\mu \nu}^{1,2}\right]\right) .
\end{aligned}
$$

The lengthier expressions for the coefficients $\mathcal{R}^{i, j, k}$ and $\mathcal{R}^{1,2,3,4}$ are provided in Appendix $\mathrm{A}$.

\section{OPE Analysis}

We want to compute the leading contribution to the OPE expansion of various one-loop four-point correlators. That is, we consider the double coincidence limit (OPE limit) $\left|x_{12}\right|,\left|x_{34}\right| \ll\left|x_{23}\right|$, or equivalently $s \rightarrow 0, t \rightarrow 1$. In terms of the variables $z, \bar{z}$, the limit is attained for $|z| \rightarrow 0$, upon which the one-loop box integral (4.6) expands to

$$
I^{(1)}(s, t)=\log (s)-2+\mathcal{O}(\sqrt{s}) .
$$

In the following, we will focus on the leading term $\log (s)$ only. In the OPE decomposition, this term is completely captured by the anomalous dimensions of the internal operators: For an operator with scaling dimension

$$
\Delta=\Delta_{0}+\lambda \Delta_{1}+\mathcal{O}\left(\lambda^{2}\right)
$$

the two-point function expands to

$$
\frac{1}{x^{2 \Delta}}=\frac{1}{x^{2 \Delta_{0}}}\left(1-2 \lambda \Delta_{1} \log |x|\right)+\mathcal{O}\left(\lambda^{2}\right) .
$$

Considering the OPE sum of any four-point correlator,

$$
\left\langle\mathcal{O}_{1}\left(x_{1}\right) \mathcal{O}_{2}\left(x_{2}\right) \mathcal{O}_{3}\left(x_{3}\right) \mathcal{O}_{4}\left(x_{4}\right)\right\rangle=\sum_{M} C_{12}^{M}\left(x_{12}\right)\left\langle\mathcal{O}_{M}\left(x_{2}\right) \mathcal{O}_{M}\left(x_{3}\right)\right\rangle C_{34}^{M}\left(x_{34}\right),
$$

it is clear that, at one-loop order, every operator $\mathcal{O}_{M}$ with a non-vanishing anomalous dimension will contribute a $\log \left|x_{23}\right|$ term. Now, the double coincidence limit is equivalent to letting $\left|x_{23}\right| \rightarrow \infty$, which implies the identification

$$
\log (s)=-4 \log \left|x_{23}\right|
$$


Moreover, since $\left\langle\mathcal{O}_{M}\left(x_{2}\right) \mathcal{O}_{M}\left(x_{3}\right)\right\rangle \sim\left|x_{23}\right|^{-\Delta_{0}}$, the leading term in the OPE sum in the $\left|x_{23}\right| \rightarrow \infty$ limit stems from operators $\mathcal{O}_{M}$ with lowest classical dimension $\Delta_{0}$. Combining $(5.3),(5.4)$, and $(5.5)$, one finds that the leading $\log (s)$ part of any one-loop four-point correlator is given by

$$
\begin{aligned}
&\left\langle\mathcal{O}_{1}\left(x_{1}\right) \mathcal{O}_{2}\left(x_{2}\right) \mathcal{O}_{3}\left(x_{3}\right)\right.\left.\mathcal{O}_{4}\left(x_{4}\right)\right\rangle^{\text {one-loop }}= \\
& \frac{\lambda}{2} \log (s) \sum_{M} \Delta_{M, 1}\left(C_{12}^{M}\left(x_{12}\right)\left\langle\mathcal{O}_{M}\left(x_{2}\right) \mathcal{O}_{M}\left(x_{3}\right)\right\rangle C_{34}^{M}\left(x_{34}\right)\right)^{\text {class. }}+\ldots
\end{aligned}
$$

where the sum runs over operators $\mathcal{O}_{M}$ of the smallest classical dimension $\Delta_{0}$ that have a non-vanishing anomalous dimension $\Delta_{1}$, "class." stands for the classical part, and the ellipsis stands for terms that are power-suppressed in $s$ or $\log (s)$.

Specializing to operators made of the twisted fields $\phi(x), V_{\mu}(x)$, and $B(x)$, the operators with lowest twisted scaling dimension and non-vanishing anomalous dimension are exactly the two-magnon states (2.18), and therefore the sum in (5.6) runs over exactly those states. Below, we will compute the OPE sum for several cases.

The full two-magnon supermultiplet comprises 13 types of states [11]. Four of those have only fermionic excitations, and since we will only consider bosonic excitations on the external states, their classical OPE coefficients will always vanish. Hence we can neglect these types of states. Another three types of states are antisymmetric under exchange of their two excitations. Since we will not consider antisymmetric excitations on the external states, these types of operators can also be neglected. What remains are the six types of operators with bosonic excitations listed in (2.18).

In the OPE expansion of four scalar operators of length $k$, generically all states with lengths $L=\{2, \ldots, 2 k\}$ contribute. Of the six types of operators in the two-magnon supermultiplet (2.18), three types have length $L=J+2$, while the other three types have length $L=J+4$. At a given length $L$, six types of operators contribute; they belong to two different supermultiplets with $J=L-2$ and $J=L-4$. Generically, complete multiplets contribute to the OPE. The exception is the extremal case $L=2 k$, where only one half of the multiplet with $J=L-2$ contributes.

In the following, we will compute the OPE sum of the right-hand side of (5.6) for several example correlators, using the OPE coefficients obtained in Section 3. An overview of the results is shown in Table 1 . In all cases, we find that the double-coincidence limit of the known four-point correlators (Section 4) is correctly reproduced by the OPE sum over complete two-magnon supermultiplets. The operators of the extremal half-multiplet at $J=2 k-2=L-2$ produce a spurious contribution that has to be canceled by double-trace states, on which we comment in Section 5.7. Unless the whole four-point correlator vanishes, the spurious terms are suppressed in the limit of large weight $k$.

\subsection{Four Vacua}

We start with the correlator of four vacuum states. It is easy to see from the two-point functions (3.3) and OPE coefficients (3.10) that the contributions of the operators $\mathcal{O}_{p}^{J}, \mathcal{O}_{p,(\mu \nu)}^{J, \partial \partial}$, and $\mathcal{O}_{p, \mu \nu}^{J, V \partial, 1}$ as well as the contributions of the operators $\mathcal{O}_{p}^{J, \partial \partial}, \mathcal{O}_{p,(\mu \nu)}^{J, V V}$, and $\mathcal{O}_{p, \mu \nu}^{J, V \partial, 2}$ separately sum up to zero. Hence the OPE limit vanishes at one-loop order. This is expected, since the vacuum correlator $\left\langle\mathcal{Q}_{1} \mathcal{Q}_{2} \mathcal{Q}_{3} \mathcal{Q}_{4}\right\rangle$ is protected. 


\begin{tabular}{ccccc} 
Correlator \& & $\begin{array}{c}\text { Correlator } \\
\text { OPE channel }\end{array}$ & $\begin{array}{c}\text { Match with full } \\
\text { expression }\end{array}$ & $\begin{array}{c}\text { Remainder } \\
\text { srom half SM }\end{array}$ & $\begin{array}{c}\text { Tensor structure } \\
\text { from single SM }\end{array}$ \\
\hline & 0 & $\checkmark$ & 0 & $\checkmark$ \\
\hline
\end{tabular}

Table 1: Overview of results of the OPE analysis for the various four-point correlators. Black dots stand for vacuum operators $\mathcal{Q}$, white dots stand for excited operators $\mathcal{Q}_{\mu}$. Column 3 indicates the matching of the OPE sum over full two-magnon multiplets (with $0 \leq J \leq 2 k-4$ ) with the OPE limit of the known one-loop correlator (column 2). Column 4 lists references to the spurious remainders from extremal multiplets (with $J=2 k-2$ ). The last column indicates whether the OPE sum over a single supermultiplet already produces the right tensor structure of the correlator.

\subsection{One Excitation}

Next, consider the four-point correlator of one single-excitation operator with three vacuum operators, $\left\langle\mathcal{Q}_{\mu, 1} \mathcal{Q}_{2} \mathcal{Q}_{3} \mathcal{Q}_{4}\right\rangle$. For every $J$ and $p$, using the two-point functions (3.3) and the OPE coefficients $(3.10,3.13)$, one finds that the OPE expansion can be split into a part proportional to $(2 k-L)$ and a remainder. All pieces proportional to $(2 k-L)$ cancel among states with $L=J+2$, and similarly among states with $L=J+4$ within a single multiplet. The remaining terms also cancel within a single multiplet, but in combinations of terms with $L=J+2$ and $L=J+4$. As a result, each full supermultiplet contributes zero. This is the expected result, since the full one-excitation correlator is protected (4.12). But the extremal case $L=2 k$ produces a remainder, from the half-multiplet with $J=L-2=2 k-2$. The contribution of this remainder to the classical OPE reads

$$
\sum_{p}\left(\mathcal{C}_{p}^{2 k-2}\right)^{2} 2^{3 / 2} k^{3} x_{12}^{\alpha}\left(-I_{23, \mu \beta} I_{23, \alpha \gamma}+\frac{1}{2} \eta_{\mu \alpha} \eta_{\beta \gamma}\right) x_{23}^{-4} x_{34}^{\beta} x_{34}^{\gamma} .
$$

Adding the one-loop anomalous dimension (2.20), the sum over momenta gives

$$
\sum_{p}\left(\mathcal{C}_{p}^{2 k-2}\right)^{2} \Delta_{1}(p)=\frac{L}{8 \pi^{2}}=\frac{J+2}{8 \pi^{2}}=\frac{k}{4 \pi^{2}} .
$$

Hence, the contributions of the extremal half-multiplets sum to

$$
\frac{\lambda}{2} \log (s) \frac{k^{4}}{\sqrt{2} \pi^{2}} x_{12}^{\alpha}\left(-I_{23, \mu \beta} I_{23, \alpha \gamma}+\frac{1}{2} \eta_{\mu \alpha} \eta_{\beta \gamma}\right) x_{23}^{-4} x_{34}^{\beta} x_{34}^{\gamma} .
$$

Since the one-excitation correlator vanishes (4.12), this result needs to be canceled by another contribution. At leading order in $1 /\left|x_{23}\right|$, the supermultiplets $(2.18)$ exhaust the single-trace contributions, but it is well-known that extremal states with $L=2 k$ mix with double-trace operators. Adding the contribution of those operators should cancel the term (5.9). See the discussion in Section 5.7 below. 


\subsection{Two Excitations on One Side}

Combining the OPE coefficients (3.13) and (3.15) with the two-point functions $(3.3)$, one finds that the contributions of individual operators to the OPE of the correlator $\left\langle\mathcal{Q}_{\mu, 1} \mathcal{Q}_{\nu, 2} \mathcal{Q}_{3} \mathcal{Q}_{4}\right\rangle$ are quadratic in $(2 k-L)$. The sum of contributions within each half-multiplet (with $L=J+2$ or $L=J+4)$ is only linear in $(2 k-L)$, the quadratic piece cancels. In the sum over each full supermultiplet, all dependence on $(2 k-L)$ cancels (except for overall factor $\left.\left(\mathcal{C}_{p}^{J}\right)^{2} k^{2}\right)$. The contribution of all full supermultiplets to the OPE reads

$$
\sum_{\substack{J=0 \\ J \text { even }}}^{2 k-4} \sum_{p}-\left(\mathcal{C}_{p}^{J}\right)^{2} 2 k^{2}\left(I_{12, \mu \gamma} I_{23}{ }^{\gamma}{ }_{\alpha} I_{23, \nu \beta}+\frac{x_{12, \mu} x_{12, \nu}}{x_{12}^{2}} \eta_{\alpha \beta}+(\mu \leftrightarrow \nu)\right) x_{23}^{-4} x_{34}^{\alpha} x_{34}^{\beta} .
$$

Adding the anomalous dimension, the sum over multiplets (momenta $p$ and weights $J$ ) gives

$$
\sum_{\substack{J=0 \\ J \text { even }}}^{2 k-4} \sum_{p}\left(\mathcal{C}_{p}^{J}\right)^{2} \Delta_{1}(p)=\frac{k(k-1)}{8 \pi^{2}}
$$

Hence the contributions of all complete two-magnon supermultiplets to the one-loop four-point correlator sum to

$$
\frac{\lambda}{2} \log (s) \frac{k^{3}(k-1)}{8 \pi^{2}}\left[-2\left(I_{12, \mu \gamma} I_{23}{ }_{\alpha}{ }_{\alpha} I_{23, \nu \beta}+\frac{x_{12, \mu} x_{12, \nu}}{x_{12}^{2}} \eta_{\alpha \beta}+(\mu \leftrightarrow \nu)\right) x_{23}^{-4} x_{34}^{\alpha} x_{34}^{\beta}\right] .
$$

The term in the square brackets exactly equals the prefactor $\mathcal{R}_{\mu \nu}^{1,2}(\mathrm{~A} .12)$ in the double-coincidence limit $\left|x_{12}\right|,\left|x_{34}\right| \ll\left|x_{23}\right|$. Comparing with (4.13), we therefore find that (5.12) reproduces the limit of the known four-point correlator.

However, there is also the extremal half supermultiplet at $L=2 k=J+2$, which gives the following non-trivial contribution to the classical OPE:

$$
\sum_{p}-\left(\mathcal{C}_{p}^{2 k-2}\right)^{2} k^{2} \frac{\eta_{\mu \nu} x_{34}^{2}}{x_{23}^{4}}
$$

Adding the anomalous dimension $\Delta_{1}(p)$ and summing over momenta $(\overline{5.8})$ results in the following extra contribution:

$$
\frac{\lambda}{2} \log (s)\left(-\frac{k^{3}}{4 \pi^{2}} \frac{\eta_{\mu \nu} x_{34}^{2}}{x_{23}^{4}}\right) .
$$

Again, this should be canceled by contributions from the relevant double-trace operators.

\subsection{One Excitation on Each Side}

Next, consider the correlator $\left\langle\mathcal{Q}_{\mu, 1} \mathcal{Q}_{2} \mathcal{Q}_{3} \mathcal{Q}_{\sigma, 4}\right\rangle$. The analysis parallels the previous case. Again, each individual operator contribution to the OPE is quadratic in $(2 k-L)$, the contribution of each half-multiplet is linear in $(2 k-L)$, and the contribution of each full supermultiplet is independent of $(2 k-L)$. The contributions from full supermultiplets to the classical OPE give

$$
\sum_{\substack{J=0 \\ J \text { even }}}^{2 k-4} \sum_{p}-\left(\mathcal{C}_{p}^{J}\right)^{2} 4 k^{2} x_{12}^{\alpha}\left(\frac{1}{2} I_{23, \alpha \beta}\left(I_{12, \mu \gamma} I_{23}{ }^{\gamma} \sigma+I_{23, \mu \gamma} I_{34}{ }^{\gamma} \sigma-2 I_{23, \mu \sigma}\right)+I_{23, \mu \beta} I_{23, \sigma \alpha}+\eta_{\mu \alpha} \eta_{\sigma \beta}\right) x_{23}^{-4} x_{34}^{\beta} .
$$


Inserting the one-loop anomalous dimension and performing the state sums yields

$$
\begin{aligned}
\frac{\lambda}{2} \log (s) \frac{k^{3}(k-1)}{8 \pi^{2}}\left[-4 x_{12}^{\alpha}\left(\frac{1}{2} I_{23, \alpha \beta}\left(I_{12, \mu \gamma} I_{23}{ }^{\gamma}{ }_{\sigma}+I_{23, \mu \gamma} I_{34}{ }^{\gamma}{ }-2 I_{23, \mu \sigma}\right)\right.\right. & \\
& \left.\left.+I_{23, \mu \beta} I_{23, \sigma \alpha}+\eta_{\mu \alpha} \eta_{\sigma \beta}\right) x_{23}^{-4} x_{34}^{\beta}\right]
\end{aligned}
$$

for the one-loop OPE. The expression in the square brackets equals the leading part of $\mathcal{R}_{\mu \sigma}^{1,4}(\mathrm{~A} .15)$ in the double-coincidence limit $\left|x_{12}\right|,\left|x_{34}\right| \ll\left|x_{23}\right|$, and hence (5.16) equals the leading part of the known correlator $(4.14)$ in that limit.

Again, there is an extremal half-multiplet at $2 k=L=J+2$, which produces the following contribution to the classical OPE:

$$
\sum_{p}\left(\mathcal{C}_{p}^{2 k-2}\right)^{2} 2 k^{2} x_{12}^{\alpha}\left(I_{23, \mu \sigma} I_{23, \alpha \beta}-\eta_{\mu \alpha} \eta_{\sigma \beta}\right) x_{23}^{-4} x_{34}^{\beta} .
$$

Via (5.8), this gives rise to the spurious contribution

$$
\frac{\lambda}{2} \log (s) \frac{k^{3}}{2 \pi^{2}} x_{12}^{\alpha}\left(I_{23, \mu \sigma} I_{23, \alpha \beta}-\eta_{\mu \alpha} \eta_{\sigma \beta}\right) x_{23}^{-4} x_{34}^{\beta}
$$

to the one-loop OPE. Again, this should be canceled by the relevant double-trace contributions.

\subsection{Three Excitations}

In the case of the three-excitation correlator $\left\langle\mathcal{Q}_{\mu, 1} \mathcal{Q}_{\nu, 2} \mathcal{Q}_{3} \mathcal{Q}_{\sigma, 4}\right\rangle$, summing over all contributions of a single supermultiplet yields a lengthy expression. In the contributions of full supermultiplets to the classical OPE

$$
\sum_{\substack{J=0 \\ J \text { even }}}^{2 k-4} \sum_{p}\left(\mathcal{C}_{p}^{J}\right)^{2} k^{2}(\cdots)
$$

the term in parantheses can be constructed from the OPE coefficients $(3.13,3.15)$ and two-point functions (3.3). It is a long expression that depends both on $k$ (quadratically) and $J$ (linearly). Nonetheless, inserting the one-loop anomalous dimension $\Delta_{1}$ and performing the sums over momenta $p$ and charges $J$ exactly yields the expected expression (4.17).

As in the previous cases, the extremal half supermultiplet contributes a spurious remainder, which takes the surprisingly simple form

$$
\frac{\lambda}{2} \log (s) \sum_{p}\left(\mathcal{C}_{p}^{2 k-2}\right)^{2} \Delta_{1}(p)(\ldots)=\frac{\lambda}{2} \log (s) \frac{k^{2}}{2^{3 / 2} \pi^{2}} \frac{\eta_{\mu \nu} x_{34, \sigma}}{x_{23}^{4}},
$$

and wich should be canceled by the relevant double-trace contributions.

\subsection{Four Excitations}

The contributions to the OPE of the four-excitation correlator $\left\langle\mathcal{Q}_{\mu, 1} \mathcal{Q}_{\nu, 2} \mathcal{Q}_{\rho, 3} \mathcal{Q}_{\sigma, 4}\right\rangle$ are yet more complicated functions. In the sum over full supermultiplets

$$
\sum_{\substack{J=0 \\ J \text { even }}}^{2 k-4} \sum_{p}\left(\mathcal{C}_{p}^{J}\right)^{2} k(\ldots)
$$


the term in parentheses is again a long expression (constructed from $(3.15,3.3)$ ) that depends quadratically on both $k$ and $J$. Yet, performing the sums yields exactly the wanted result (4.18). Also in this case, the spurious term from the extremal half supermultiplet at $J=2 k-2$ is remarkably simple:

$$
\frac{\lambda}{2} \log (s) \sum_{p}\left(\mathcal{C}_{p}^{2 k-2}\right)^{2} \Delta_{1}(p)(\ldots)=\frac{\lambda}{2} \log (s) \frac{k}{4 \pi^{2}} \frac{\eta_{\mu \nu} \eta_{\rho \sigma}}{x_{23}^{4}}
$$

Again, this term should be canceled by contributions from double-trace operators.

\subsection{Note on Double-Trace Operators}

In all examples above, we have seen that the leading term in the double-coincidence limit of the one-loop correlator is correctly reproduced by summing over complete two-magnon supermultiplets in the OPE channel, neglecting the extremal half-supermultiplet with charge $J=2 k-2$. Including the extremal half-multiplet in the OPE sum leads to a spurious contribution that needs to be canceled by further operators. Since the two-magnon operators $(2.18)$ exhaust the single-trace spectrum at $\hat{\Delta}_{0}=2$ and $\Delta_{1} \neq 0$, those further states have to be multi-trace states. In fact, it is well-known that single-trace and double-trace operators mix at subleading order in $1 / N_{\mathrm{c}}[19-21]$. That is, single-trace operators obtain double-trace corrections that are suppressed by a factor of $1 / N_{\mathrm{c}}$. In extremal three-point functions, each trace of the doubletrace operator can separately contract with the two other external operators. Such terms are enhanced by a factor $N_{\mathrm{c}}$ that cancels the $1 / N_{\mathrm{c}}$ suppression. Hence they affect extremal three-point functions at leading order in $1 / N_{\mathrm{c}}$. The double-trace corrections to our two-magnon states $(2.18)$ carry two excitations as well. There are three cases to distinguish:

(i) both excitations sit on the same trace factor, with a non-zero momentum,

(ii) both excitations sit on the same trace factor, with zero momentum,

(iii) each of the two trace factors carries one of the two excitations.

In the first case, the excited trace factor is itself one of the two-magnon states (2.18). In case (ii) and (iii), each of the excited trace factors is a descendant of the vacuum operator. Two-magnon states with non-zero momentum contract to zero with the external operators considered above, hence corrections of type (i) do not contribute to the OPE. We are thus looking for a double-trace correction of type (ii) or (iii) to a two-magnon state (2.18). Such corrections do indeed exist: For example, the singlet state $\mathcal{O}_{p}^{J}$ gets a correction of type (iii) (see equation (3.27) in $[20 \mid$ ).

There are further contributions to the OPE due to double-trace operators: While doubletrace operators of type (ii) and (iii) have vanishing anomalous dimension at leading order in the planar limit, they acquire single-trace corrections at subleading order in $1 / N_{\mathrm{c}}$, which can lead to $1 / N_{\mathrm{c}}^{2}$ corrections to their anomalous dimensions. Combined with the $N_{\mathrm{c}}$ enhancement of extremal three-point functions with double-trace operators, also these corrections can affect the four-point OPE at leading order in $1 / N_{\mathrm{c}}$. Indeed, double-trace operators of type (iii) get corrected by two-magnon states with non-zero momenta (see equation (3.16) in $[20]$ ), which shows that this effect indeed occurs.

Our results show that the contributions from extremal states at charge $J=2 k-2$ need to be canceled by double-trace contributions of the type described above. On a technical level, computing all such double-trace contributions explicitly is not entirely straightforward, because the orthogonalization procedure for zero-momentum states is non-trivial already in the untwisted 
case [20], and is further complicated by the twisting. Nonetheless, it would be desirable to compute the double-trace contributions in order to confirm their cancellation. Here, we take the standpoint that the extremal contributions $(5.9,5.14,5.18,5.20,5.22)$ provide predictions for the sum of all double-trace contributions to the various OPEs.

\section{Conclusions}

We have considered four-point correlators of single-trace scalar operators that are organized in an excitation picture around specific vacuum operators. Both the vacuum operators and the excitations are related to the familiar $\mathfrak{p s u}(2,2 \mid 4)$ spin chain picture via a twisting procedure that ties the orientation of the vacuum in the internal $S^{5}$ to the spacetime location. The motivation for this choice of operators is that any number of such operators is preserved by two common supercharges $\mathfrak{Q}^{ \pm}$, and that all other operators arrange themselves in terms of excitations on top of the vacuum operators that fall in representations of a common $\mathfrak{q}(3)$ symmetry algebra. These properties make the twisted operators promising candidates for an integrability-based treatment of higher-point correlation functions.

In this study, we have barely scratched the surface of these correlation functions: We have only considered zero-momentum excitations, and in the OPE decomposition, we have restricted ourselves to the leading non-trivial terms. Nevertheless, we can make a few non-trivial observations:

For every correlator with up to two excitations that we considered, we find that each complete two-magnon supermultiplet separately contributes the correct tensor structure to the one-loop correlator in the double-coincident limit. In particular, for protected correlators, the contributions from each two-magnon supermultiplet separately sum up to zero. This appears to be a non-trivial fact. It could as well have been that the correct tensor structure is only obtained after summing over multiplets.

For every correlator that we considered, performing the sum over complete two-magnon multiplets (momenta $p$ and charges $J \leq 2 k-4$ ) exactly reproduces the OPE limits of the known four-point correlators. In particular, this sum over complete supermultiplets does not include states from the multiplet at "extremal" charge $J=2 k-2$, even though these states $d o$ contribute non-trivially to the OPE. The fact that those states need to be discarded in order to recover the known correlators shows that their contributions need to be canceled by further states. Since the two-magnon states we considered are the only single-trace states that can contribute at leading order in the double-coincidence limit, those further states have to be double-trace states. These are known to undergo a $1 / N_{\mathrm{c}}$ mixing with single-trace operators in extremal OPE coefficients (three-point functions), and thus do contribute to the OPE at leading order in $1 / N_{\mathrm{c}}$. Even though we have not computed the double-trace contributions, our results show that they have to cancel the contributions of the extremal single-trace supermultiplet at $J=2 k-2$. Turning the logic around, our results constitute predictions for the double-trace contributions to the various OPEs.

In general, four- and higher-point functions have an intricate spacetime dependence, both through complicated tensor structures and through non-trivial functional dependence on conformally invariant cross ratios. Perhaps one of the strongest advantages of the twisted correlators is that all spacetime dependence is directly coupled to the magnons (excitations) on the external operators. It can be seen directly from the contraction rules (3.1) that inserting excitations on top of the vacuum operators injects spacetime factors into the free-field contractions. Correspondingly, the respective spacetime factors emerge from the general formula $(4.1)$ for half-BPS operators, as in the examples $(4.13),(4.14),(4.17)$, and $(4.18)$. 
The OPE of vacuum operators $\operatorname{tr}[\phi \ldots \phi]$ alone already is an interesting object. The set of vacuum operators is not closed under the OPE, as exemplified by the non-trivial OPE coefficients (3.10) with two-magnon states considered here. Since all correlation functions of vacuum operators are protected, and evaluate to constants, this implies non-trivial cancellations among all operators that flow in the OPE.

\section{Outlook}

We have only taken the very first steps in the study of higher-point correlation functions of twisted operators, which leaves a lot of room for further explorations. The possibilities include:

It would be very interesting to see whether extremal and double-trace contributions continue to cancel out also for more general operators (with non-zero-momentum excitations), and beyond leading order in the double-coincidence limit. If this holds, it would mean that one could entirely avoid to resolve the mixing with double-trace operators. To this end, it would be interesting to compute the double-trace contributions explicitly for some examples.

It would be very desirable to promote the heuristic results presented here to a more systematic description of four-point (and perhaps higher-point) functions, based on the excitation picture over twisted vacua. The only ingredients should be the magnon rapidities and flavors of the excitations on the four external operators, the two-magnon S-matrix, as well as general symmetry principles. In particular, also the spacetime dependence should be captured completely; since it is directly tied to the external magnons, it can perhaps be absorbed in a redefined two-body S-matrix.

In order to understand the above points, one should obviously extend the present analysis beyond half-BPS operators by including excitations with non-zero momenta on the external states.

Excitations on top of the vacuum operators $\operatorname{tr}[\phi \ldots \phi]$ organize in representations of a common $\mathfrak{q}(3)$ symmetry algebra. It would be very interesting to study the consequences of this symmetry.

We have used the lowest-lying non-protected states to reconstruct the leading logarithmic term of the one-loop correlators in the double-coincidence limit. It would be interesting to see to what extent this procedure could be generated to higher orders, both in kinematics and in the coupling. Assuming that a given correlator can be expanded in a suitable integral basis, such a procedure could produce constraints on the expansion coefficients. In the expansion of four-point correlators around the double-coincidence limit, the leading term will always be given by the two-magnon operators $(2.18)$, since these are the states with lowest classical twisted dimension among all operators with non-trivial anomalous dimension. Discerning contributions from different basis integrals at higher loops will require to include terms at subleading order, which are provided by internal operators with more than two excitations (and thus higher twisted dimension).

In order to construct the one-loop OPE, we had to compute three-point functions of the twisted two-magnon states with zero-momentum operators. The former are twisted versions of the two-magnon operators of [11], while the latter are twisted versions of R-symmetry descendants of the BMN vacuum $\operatorname{tr}\left[Z^{J}\right]$. Due to the twisting, the three operators span the whole scalar $\mathfrak{s o}(6)$ sector. It would be interesting to study these higher-rank three-point functions more thoroughly, and to compare them with the formulation in terms of hexagon form factors [4]. In particular, due to the uncommon representation of the zero-momentum operators under the twisted conformal symmetry (2.15), correlators involving such operators have a non-standard spacetime dependence. More generally, it would be interesting to work out the implications 
for correlation functions (Ward identities) of operators that transform non-trivially under the twisted special conformal generator $\hat{\kappa}_{\mu}$.

It would also be interesting to study the correlators of twisted operators at strong coupling. Using embedding coordinates $Y^{I}, I=1, \ldots, 6$ for $\mathrm{AdS}_{5}$, the boundary Minkowski space, parametrized by $x^{\mu}$, is mapped to the lightcone

$$
Y=\left(x^{\mu}, \frac{1}{2}\left(1+x^{2}\right), \frac{1}{2}\left(1-x^{2}\right)\right) .
$$

This shows that for the vacuum operators $\operatorname{tr}\left[\phi^{J}\right]$, the internal coordinate $X(2.2)$ is identified with the spacetime coordinate $Y$ (up to factors of $i$ due to the different signatures, and the overall normalization). This is a direct consequence of the definition via the twisted translation (2.8). In the dual sigma model on $\mathrm{AdS}_{5} \times \mathrm{S}^{5}$, setting $X=Y$ amounts to equating the coordinates on the sphere with the AdS coordinates. It would be interesting to study solutions of this "reduced" sigma model. Perhaps classical solutions corresponding to three-point or higher-point correlators can be found.

While this work was being completed, two very interesting papers [22,23] proposed a decomposition of twisted correlators (exactly of the type studied here) in terms of the hexagon form factors constructed earlier in the context of three-point functions [4]. In particular, the work [23] provides a systematic description of four-point functions (including the spacetime dependence) in terms of integrability data, without reference to the OPE. Interestingly, Fleury and Komatsu [23] observe that they need to exclude certain "one-edge reducible" graphs from their sum over propagator structures in order to reproduce the correct perturbative results. It appears plausible that this observation is related to our finding that certain extremal and all double-trace operators cancel out in the OPE sum.

Finally, let us end on a note about the fate of $\mathcal{N}=4 \mathrm{sYM}$ integrability beyond the planar limit. Higher-point correlation functions are inherently suppressed by powers of $1 / N_{\mathrm{c}}$ compared to two-point functions: Counting powers of $1 / N_{\mathrm{c}}$, four-point functions on the sphere are of the same order as two-point functions on the torus. In fact, the two are related by a complete state sum on the side of the four-point function. If indeed planar four-point functions can be computed with integrability, tackling non-planar processes by integrability techniques may finally come within reach.

\section{Acknowledgments}

I thank Niklas Beisert and Juan Maldacena for several illuminating discussions, and the IAS Princeton for support during the initial stage of this work. This work was in part supported by a Marie Curie International Outgoing Fellowship within the $7^{\text {th }}$ European Community Framework Programme under Grant No. PIOF-GA-2011-299865.

\section{A Details on Tensors}

\section{Useful Identities.}

$$
\begin{gathered}
I_{\alpha \gamma}(x) I_{\beta}^{\gamma}(x)=\eta_{\alpha \beta} \\
Y_{\mu}\left(x_{i j}, x_{i k}\right) Y^{\mu}\left(x_{i j}, x_{i k}\right)=\frac{x_{j k}^{2}}{x_{i j}^{2} x_{i k}^{2}} \\
I_{\rho}{ }^{\sigma}\left(x_{i j}\right) Y_{\sigma}\left(x_{i j}, x_{i k}\right)=\frac{x_{j k}^{2}}{x_{i k}^{2}} Y_{\rho}\left(x_{j i}, x_{j k}\right)
\end{gathered}
$$


Coefficients of the Universal Prefactor. Using the shorthand notation

$$
\check{x}=\frac{x}{x^{2}},
$$

the coefficient $\mathcal{R}^{1,2}(4.15)$ reads

$$
\begin{aligned}
\mathcal{R}^{1,2, \mu \nu}=4 \check{x}_{13}^{\mu} \check{x}_{24}^{\nu}- & 4 s \check{x}_{12}^{\mu} \check{x}_{12}^{\nu}+4 t \check{x}_{14}^{\mu} \check{x}_{23}^{\nu}+2(-1+s+t)\left(\check{x}_{12}^{\nu} \check{x}_{14}^{\mu}-\check{x}_{12}^{\mu} \check{x}_{23}^{\nu}\right) \\
& +2(1+s-t)\left(\check{x}_{12}^{\nu} \check{x}_{13}^{\mu}-\check{x}_{12}^{\mu} \check{x}_{24}^{\nu}\right)+2(-1+s-t)\left(\check{x}_{13}^{\mu} \check{x}_{23}^{\nu}+\check{x}_{14}^{\mu} \check{x}_{24}^{\nu}\right) .
\end{aligned}
$$

Similarly,

$$
\begin{aligned}
\mathcal{R}^{1,4, \mu \sigma}=-4 \check{x}_{13}^{\mu} \check{x}_{24}^{\sigma}-4 s \check{x}_{12}^{\mu} \check{x}_{34}^{\sigma}-4 t \check{x}_{14}^{\mu} \check{x}_{14}^{\sigma}+2(-1+s+t)\left(\check{x}_{12}^{\mu} \check{x}_{14}^{\sigma}+\check{x}_{14}^{\mu} \check{x}_{34}^{\sigma}\right) \\
+2(1+s-t)\left(\check{x}_{12}^{\mu} \check{x}_{24}^{\sigma}+\check{x}_{13}^{\mu} \check{x}_{34}^{\sigma}\right)-2(-1+s-t)\left(\check{x}_{13}^{\mu} \check{x}_{14}^{\sigma}+\check{x}_{14}^{\mu} \check{x}_{24}^{\sigma}\right) .
\end{aligned}
$$

In fact, all $\mathcal{R}^{i, j}$ can be obtained from $\mathcal{R}^{1,2}$ by applying the following replacements:

$$
\begin{array}{ll}
\mathcal{R}^{1,3, \mu \rho}: & \check{x}_{12}^{\nu} \rightarrow-\check{x}_{34}^{\rho}, \check{x}_{23}^{\nu} \rightarrow-\check{x}_{23}^{\rho}, \check{x}_{24}^{\nu} \rightarrow-\check{x}_{13}^{\rho} \\
\mathcal{R}^{1,4, \mu \sigma}: & \check{x}_{12}^{\nu} \rightarrow+\check{x}_{34}^{\sigma}, \check{x}_{23}^{\nu} \rightarrow-\check{x}_{14}^{\sigma}, \check{x}_{24}^{\nu} \rightarrow-\check{x}_{24}^{\sigma} \\
\mathcal{R}^{2,3, \nu \rho}: & \check{x}_{12}^{\mu} \rightarrow+\check{x}_{34}^{\rho}, \check{x}_{13}^{\mu} \rightarrow-\check{x}_{13}^{\rho}, \check{x}_{14}^{\mu} \rightarrow-\check{x}_{23}^{\rho} \\
\mathcal{R}^{2,4, \nu \sigma}: & \check{x}_{12}^{\mu} \rightarrow-\check{x}_{34}^{\sigma}, \check{x}_{13}^{\mu} \rightarrow-\check{x}_{24}^{\sigma}, \check{x}_{14}^{\mu} \rightarrow-\check{x}_{14}^{\sigma} \\
\mathcal{R}^{3,4, \rho \sigma}: & \text { combinations of replacements for } \mathcal{R}^{1,3, \mu \rho} \text { and } \mathcal{R}^{2,4, \nu \sigma} \\
& \text { (or combinations of replacements for } \mathcal{R}^{1,4, \mu \sigma} \text { and } \mathcal{R}^{2,3, \nu \rho} \text {.) }
\end{array}
$$

In the double-coincidence limit $\left|x_{12}\right|\left|x_{34}\right| \ll\left|x_{23}\right|$, the coefficients $\mathcal{R}^{i, j}$ become

$$
\begin{aligned}
& \mathcal{R}_{\mu \nu}^{1,2}=-2\left(I_{12, \mu \gamma} I_{23}{ }^{\gamma}{ }_{\alpha} I_{23, \nu \beta}+x_{12, \mu} \check{x}_{12, \nu} \eta_{\alpha \beta}+(\mu \leftrightarrow \nu)\right) x_{23}^{-4} x_{34}^{\alpha} x_{34}^{\beta}, \\
& \mathcal{R}_{\mu \nu}^{3,4}=-2\left(I_{34, \mu \gamma} I_{23}{ }^{\gamma}{ }_{\alpha} I_{23, \nu \beta}+x_{34, \mu} \check{x}_{34, \nu} \eta_{\alpha \beta}+(\mu \leftrightarrow \nu)\right) x_{23}^{-4} x_{12}^{\alpha} x_{12}^{\beta}, \\
& \mathcal{R}_{\mu \rho}^{1,3}=4 x_{12}^{\alpha}\left(\frac{1}{2} I_{23, \alpha \beta}\left(I_{12, \mu \gamma} I_{23}{ }^{\gamma}{ }_{\rho}+I_{23, \mu \gamma} I_{34}{ }^{\gamma}{ }_{\rho}-2 I_{23, \mu \rho}\right)+I_{23, \mu \beta} I_{23, \rho \alpha}+\eta_{\mu \alpha} \eta_{\rho \beta}\right) x_{23}^{-4} x_{34}^{\beta}, \\
& \mathcal{R}_{\mu \sigma}^{1,4}=\mathcal{R}_{\mu \sigma}^{2,3}=-\mathcal{R}_{\mu \sigma}^{2,4}=-\mathcal{R}_{\mu \sigma}^{1,3} .
\end{aligned}
$$

Next, the coefficients with three indices:

$$
\begin{aligned}
& \mathcal{R}^{1,2,3, \mu \nu \rho}= \\
& \sqrt{2}\left[2 t \check{x}_{14}^{\mu}\left(-\eta^{\rho \nu} / x_{23}^{2}-4 \check{x}_{23}^{\rho} \check{x}_{23}^{\nu}\right)-2 \check{x}_{24}^{\nu}\left(\eta^{\rho \mu} / x_{13}^{2}+4 \check{x}_{13}^{\rho} \check{x}_{13}^{\mu}\right)+2 s \check{x}_{34}^{\rho}\left(-\eta^{\mu \nu} / x_{12}^{2}-4 \check{x}_{12}^{\mu} \check{x}_{12}^{\nu}\right)\right. \\
& \\
&+(-1+s+t)\left(\eta^{\rho \nu} \check{x}_{12}^{\mu} / x_{23}^{2}-\eta^{\mu \nu} \check{x}_{23}^{\rho} / x_{12}^{2}-2 \check{x}_{12}^{\nu} \check{x}_{14}^{\mu} Y_{23,34}^{\rho}-2 \check{x}_{23}^{\nu} \check{x}_{34}^{\rho} Y_{12,41}^{\mu}\right) \\
&+(1+s-t)\left(-\eta^{\rho \mu} \check{x}_{12}^{\nu} / x_{13}^{2}-\eta^{\mu \nu} \check{x}_{13}^{\rho} / x_{12}^{2}+2 \check{x}_{12}^{\mu} \check{x}_{24}^{\nu} Y_{13,34}^{\rho}+2 \check{x}_{13}^{\mu} \check{x}_{34}^{\rho} Y_{12,24}^{\nu}\right) \\
&\left.+(-1+s-t)\left(-\eta^{\rho \nu} \check{x}_{13}^{\mu} / x_{23}^{2}-\eta^{\rho \mu} \check{x}_{23}^{\nu} / x_{13}^{2}-2 \check{x}_{23}^{\rho} \check{x}_{24}^{\nu} Y_{13,41}^{\mu}-2 \check{x}_{13}^{\rho} \check{x}_{14}^{\mu} Y_{23,42}^{\nu}\right)\right]
\end{aligned}
$$

The coefficient $\mathcal{R}^{1,2,4, \mu \nu \rho}$ is obtained from $\mathcal{R}^{1,2,3, \nu \mu \rho}$ (note the permuted indices) by the replacements:

$$
\check{x}_{13} \leftrightarrow \check{x}_{24}, \quad \check{x}_{14} \leftrightarrow \check{x}_{23}, \quad x_{34} \rightarrow-x_{34}, \quad x_{12} \rightarrow-x_{12}, \quad \frac{\eta_{\rho \mu}}{x_{13}^{2}} \rightarrow \frac{\eta_{\rho \mu}}{x_{24}^{2}}, \quad \frac{\eta_{\rho \nu}}{x_{23}^{2}} \rightarrow \frac{\eta_{\rho \nu}}{x_{14}^{2}} .
$$


The coefficient $\mathcal{R}^{1,3,4, \mu \rho \sigma}$ is obtained from $\mathcal{R}^{1,2,3, \rho \sigma \mu}$ (note the permuted indices) by the replacements:

$$
\check{x}_{12} \leftrightarrow \check{x}_{34}, \quad \check{x}_{14} \leftrightarrow-\check{x}_{23}, \quad x_{24} \rightarrow-x_{24}, \quad x_{13} \rightarrow-x_{13}, \quad \frac{\eta_{\rho \sigma}}{x_{12}^{2}} \rightarrow \frac{\eta_{\rho \sigma}}{x_{34}^{2}}, \quad \frac{\eta_{\mu \sigma}}{x_{23}^{2}} \rightarrow \frac{\eta_{\mu \sigma}}{x_{14}^{2}} .
$$

The coefficient $\mathcal{R}^{2,3,4, \nu \rho \sigma}$ is obtained from $\mathcal{R}^{1,2,3, \sigma \rho \nu}$ (note the permuted indices) by the replacements:

$$
\check{x}_{12} \leftrightarrow-\check{x}_{34}, \quad \check{x}_{13} \leftrightarrow-\check{x}_{24}, \quad x_{14} \rightarrow-x_{24}, \quad x_{23} \rightarrow-x_{23}, \quad \frac{\eta_{\nu \sigma}}{x_{13}^{2}} \rightarrow \frac{\eta_{\nu \sigma}}{x_{24}^{2}}, \quad \frac{\eta_{\rho \sigma}}{x_{12}^{2}} \rightarrow \frac{\eta_{\rho \sigma}}{x_{34}^{2}} .
$$

In the double-coincidence limit $\left|x_{12}\right|\left|x_{34}\right| \ll\left|x_{23}\right|$, the coefficients $\mathcal{R}^{i, j, k}$ become

$$
\begin{aligned}
\mathcal{R}^{1,2,3, \mu \nu \rho}=\frac{\sqrt{2}}{x_{23}^{4}}( & -x_{34}^{\rho}\left(3 \eta^{\mu \nu}-2 I_{12}^{\mu \nu}\right)-2 I_{23}^{\nu \rho} I_{23}^{\mu} \cdot x_{34} \\
& \left.\quad+x_{12} \cdot I_{23} \cdot x_{34}\left(\eta^{\mu \nu} \check{x}_{12} \cdot I_{23}^{\rho}+2 \check{x}_{12}^{\mu}\left(2 \eta^{\rho \gamma}-I_{34}^{\rho \gamma}\right) I_{23, \gamma}^{\nu}\right)\right)+(\mu \leftrightarrow \nu), \\
\mathcal{R}^{1,2,4, \mu \nu \rho}= & -\mathcal{R}^{1,2,3, \mu \nu \rho}, \\
\mathcal{R}^{1,3,4, \rho \mu \nu}=\frac{\sqrt{2}}{x_{23}^{4}}( & -x_{12}^{\rho}\left(3 \eta^{\mu \nu}-2 I_{34}^{\mu \nu}\right)-2 I_{23}^{\nu \rho} I_{23}^{\mu} \cdot x_{12} \\
\quad & \left.\quad+x_{12} \cdot I_{23} \cdot x_{34}\left(\eta^{\mu \nu} \check{x}_{34} \cdot I_{23}^{\rho}+2 \check{x}_{34}^{\mu}\left(2 \eta^{\rho \gamma}-I_{12}^{\rho \gamma}\right) I_{23, \gamma}^{\nu}\right)\right)+(\mu \leftrightarrow \nu), \\
\mathcal{R}^{2,3,4, \rho \mu \nu}= & -\mathcal{R}^{1,3,4, \rho \mu \nu} .
\end{aligned}
$$

Finally,

$$
\begin{aligned}
& \mathcal{R}^{1,2,3,4, \mu \nu \rho \sigma}=16 \check{x}_{13}^{\rho} \check{x}_{13}^{\mu} \check{x}_{24}^{\sigma} \check{x}_{24}^{\nu}+8 \check{x}_{24}^{\sigma} \check{x}_{24}^{\nu} \eta^{\rho \mu} / x_{13}^{2}+2 \eta^{\sigma \nu}\left(4 \check{x}_{13}^{\rho} \check{x}_{13}^{\mu}+\eta^{\rho \mu} / x_{13}^{2}\right) / x_{24}^{2} \\
& +(-1+s-t)\left[4 \check{x}_{13}^{\rho} \check{x}_{14}^{\mu} \check{x}_{23}^{\nu} \check{x}_{24}^{\sigma}+4 \check{x}_{13}^{\mu} \check{x}_{14}^{\sigma} \check{x}_{23}^{\rho} \check{x}_{24}^{\nu}\right. \\
& +2\left(\check{x}_{13}^{\rho} \check{x}_{23}^{\nu}+\check{x}_{13}^{\rho} \check{x}_{24}^{\nu}+\check{x}_{23}^{\rho} \check{x}_{24}^{\nu}\right) \eta^{\sigma \mu} / x_{14}^{2}+2\left(\check{x}_{13}^{\rho} \check{x}_{14}^{\mu}+\check{x}_{13}^{\mu} \check{x}_{23}^{\rho}+\check{x}_{14}^{\mu} \check{x}_{23}^{\rho}\right) \eta^{\sigma \nu} / x_{24}^{2} \\
& \left.+2\left(\check{x}_{14}^{\sigma} \check{x}_{23}^{\nu}+\check{x}_{23}^{\nu} \check{x}_{24}^{\sigma}+\check{x}_{14}^{\sigma} \check{x}_{24}^{\nu}\right) \eta^{\rho \mu} / x_{13}^{2}+2\left(\check{x}_{13}^{\mu} \check{x}_{14}^{\sigma}+\check{x}_{13}^{\mu} \check{x}_{24}^{\sigma}+\check{x}_{14}^{\mu} \check{x}_{24}^{\sigma}\right) \eta^{\rho \nu} / x_{23}^{2}\right] \\
& +(-1+s+t)\left[4 \check{x}_{12}^{\nu} \check{x}_{14}^{\mu} \check{x}_{23}^{\rho} \check{x}_{34}^{\sigma}+4 \check{x}_{12}^{\mu} \check{x}_{14}^{\sigma} \check{x}_{23}^{\nu} \check{x}_{34}^{\rho}\right. \\
& +2\left(-\check{x}_{12}^{\nu} \check{x}_{14}^{\mu}+\check{x}_{12}^{\mu} \check{x}_{23}^{\nu}+\check{x}_{14}^{\mu} \check{x}_{23}^{\nu}\right) \eta^{\sigma \rho} / x_{34}^{2}+2\left(\check{x}_{12}^{\nu} \check{x}_{23}^{\rho}-\check{x}_{12}^{\nu} \check{x}_{34}^{\rho}+\check{x}_{23}^{\nu} \check{x}_{34}^{\rho}\right) \eta^{\sigma \mu} / x_{14}^{2} \\
& \left.+2\left(-\check{x}_{12}^{\mu} \check{x}_{14}^{\sigma}-\check{x}_{12}^{\mu} \check{x}_{34}^{\sigma}-\check{x}_{14}^{\mu} \check{x}_{34}^{\sigma}\right) \eta^{\rho \nu} / x_{23}^{2}+2\left(\check{x}_{14}^{\sigma} \check{x}_{23}^{\rho}+\check{x}_{23}^{\rho} \check{x}_{34}^{\sigma}-\check{x}_{14}^{\sigma} \check{x}_{34}^{\rho}\right) \eta^{\mu \nu} / x_{12}^{2}\right] \\
& +(1+s-t)\left[-4 \check{x}_{12}^{\mu} \check{x}_{13}^{\rho} \check{x}_{24}^{\nu} \check{x}_{34}^{\sigma}-4 \check{x}_{12}^{\nu} \check{x}_{13}^{\mu} \check{x}_{24}^{\sigma} \check{x}_{34}^{\rho}\right. \\
& +2\left(-\check{x}_{12}^{\nu} \check{x}_{13}^{\mu}+\check{x}_{12}^{\mu} \check{x}_{24}^{\nu}+\check{x}_{13}^{\mu} \check{x}_{24}^{\nu}\right) \eta^{\sigma \rho} / x_{34}^{2}+2\left(-\check{x}_{12}^{\mu} \check{x}_{13}^{\rho}+\check{x}_{12}^{\mu} \check{x}_{34}^{\rho}+\check{x}_{13}^{\mu} \check{x}_{34}^{\rho}\right) \eta^{\sigma \nu} / x_{24}^{2} \\
& \left.+2\left(\check{x}_{12}^{\nu} \check{x}_{24}^{\sigma}+\check{x}_{12}^{\nu} \check{x}_{34}^{\sigma}-\check{x}_{24}^{\nu} \check{x}_{34}^{\sigma}\right) \eta^{\rho \mu} / x_{13}^{2}+2\left(\check{x}_{13}^{\rho} \check{x}_{24}^{\sigma}+\check{x}_{13}^{\rho} \check{x}_{34}^{\sigma}-\check{x}_{24}^{\sigma} \check{x}_{34}^{\rho}\right) \eta^{\mu \nu} / x_{12}^{2}\right] \\
& +s\left[16 \check{x}_{12}^{\mu} \check{x}_{12}^{\nu} \check{x}_{34}^{\sigma} \check{x}_{34}^{\rho}+8 \check{x}_{34}^{\sigma} \check{x}_{34}^{\rho} \eta^{\mu \nu} / x_{12}^{2}+2 \eta^{\sigma \rho}\left(4 \check{x}_{12}^{\mu} \check{x}_{12}^{\nu}+\eta^{\mu \nu} / x_{12}^{2}\right) / x_{34}^{2}\right] \\
& +t\left[16 \check{x}_{14}^{\sigma} \breve{x}_{14}^{\mu} \breve{x}_{23}^{\rho} \check{x}_{23}^{\nu}+8 \check{x}_{14}^{\sigma} \check{x}_{14}^{\mu} \eta^{\rho \nu} / x_{23}^{2}+2 \eta^{\sigma \mu}\left(4 \check{x}_{23}^{\rho} \check{x}_{23}^{\nu}+\eta^{\rho \nu} / x_{23}^{2}\right) / x_{14}^{2}\right] .
\end{aligned}
$$

In the double-coincidence limit, this becomes 


$$
\begin{aligned}
\mathcal{R}^{1,2,3,4, \mu \nu \rho \sigma}= & \frac{1}{x_{23}^{4}}\left(-2 x_{12} \cdot I_{23} \cdot x_{34}\left(\check{x}_{12}^{\mu} I_{23}^{\nu} \cdot \check{x}_{34} \eta^{\rho \sigma}+\eta^{\mu \nu} \check{x}_{12} \cdot I_{23}^{\rho} \check{x}_{34}^{\sigma}+2 \check{x}_{12}^{\mu} I_{23}^{\nu \rho} \breve{x}_{34}^{\sigma}\right)\right. \\
& \left.+I_{23}^{\mu \rho} I_{23}^{\nu \sigma}+\left(I_{12}^{\mu \nu}-2 \eta^{\mu \nu}\right)\left(I_{34}^{\rho \sigma}-2 \eta^{\rho \sigma}\right)-\frac{1}{2} \eta^{\mu \nu} \eta^{\rho \sigma}\right)+(\mu \leftrightarrow \nu)+(\rho \leftrightarrow \sigma)
\end{aligned}
$$

\section{References}

[1] N. Beisert et al., "Review of AdS/CFT Integrability: An Overview", Lett. Math. Phys. 99, 3 (2012), arxiv:1012.3982.

[2] N. Gromov, V. Kazakov, S. Leurent and D. Volin, "Quantum Spectral Curve for Planar $\mathcal{N}=4$ Super-Yang-Mills Theory", Phys. Rev. Lett. 112, 011602 (2014), arxiv:1305.1939 • N. Gromov, V. Kazakov, S. Leurent and D. Volin, "Quantum spectral curve for arbitrary state/operator in $A d S_{5} / C F T_{4}$ ", JHEP 1509, 187 (2015), arxiv:1405.4857.

[3] B. Basso, A. Sever and P. Vieira, "Spacetime and Flux Tube S-Matrices at Finite Coupling for $\mathcal{N}=4$ Supersymmetric Yang-Mills Theory", Phys. Rev. Lett. 111, 091602 (2013), arxiv:1303.1396 • B. Basso, A. Sever and P. Vieira, "Space-time S-matrix and Flux tube S-matrix II. Extracting and Matching Data", JHEP 1401, 008 (2014), arxiv:1306.2058

[4] B. Basso, S. Komatsu and P. Vieira, "Structure Constants and Integrable Bootstrap in Planar $\mathcal{N}=4 S Y M$ Theory", arxiv:1505.06745.

[5] B. Basso, S. Caron-Huot and A. Sever, "Adjoint BFKL at finite coupling: a short-cut from the collinear limit", JHEP 1501, 027 (2015), arxiv:1407.3766. • B. Basso, A. Sever and P. Vieira, "Hexagonal Wilson Loops in Planar $\mathcal{N}=4$ SYM Theory at Finite Coupling", J. Phys. A49, 41LT01 (2016), arxiv:1508.03045.

[6] N. Beisert, "The su(2/2) dynamic S-matrix", Adv. Theor. Math. Phys. 12, 945 (2008), hep-th/0511082

[7] P. de Medeiros, C. M. Hull, B. J. Spence and J. M. Figueroa-O'Farrill, "Conformal topological Yang-Mills theory and de Sitter holography", JHEP 0208, 055 (2002), hep-th/0111190

[8] N. Drukker and J. Plefka, "Superprotected n-point correlation functions of local operators in $\mathcal{N}=4$ super Yang-Mills", JHEP 0904, 052 (2009), arxiv:0901.3653.

[9] N. Drukker and J. Plefka, "The Structure of n-point functions of chiral primary operators in $\mathcal{N}=4$ super Yang-Mills at one-loop", JHEP 0904, 001 (2009), arxiv:0812.3341.

[10] B. Basso, F. Coronado, S. Komatsu, H. T. Lam, P. Vieira and D.-l. Zhong, "Asymptotic Four Point Functions", arxiv: 1701.04462

[11] N. Beisert, "BMN Operators and Superconformal Symmetry", Nucl. Phys. B659, 79 (2003), hep-th/0211032.

[12] P. Liendo and C. Meneghelli, "Bootstrap equations for $\mathcal{N}=4$ SYM with defects", JHEP 1701, 122 (2017), arxiv:1608.05126.

[13] C. Beem, M. Lemos, P. Liendo, W. Peelaers, L. Rastelli and B. C. van Rees, "Infinite Chiral Symmetry in Four Dimensions", Commun. Math. Phys. 336, 1359 (2015), arxiv:1312.5344.

[14] G. Mack and A. Salam, "Finite component field representations of the conformal group", Annals Phys. 53, 174 (1969)

[15] V. G. Kac, "Lie Superalgebras", Adv. Math. 26, 8 (1977)

[16] G. Arutyunov and E. Sokatchev, "On a large $N$ degeneracy in $\mathcal{N}=4 S Y M$ and the AdS/CFT correspondence", Nucl. Phys. B663, 163 (2003), hep-th/0301058 
[17] N. I. Ussyukina and A. I. Davydychev, "An Approach to the evaluation of three- and four-point ladder diagrams", Phys. Lett. B298, 363 (1993) • N. I. Ussyukina and A. I. Davydychev, "Exact results for three- and four-point ladder diagrams with an arbitrary number of rungs",

Phys. Lett. B305, 136 (1993).

[18] F. C. S. Brown, "Polylogarithmes multiples uniformes en une variable", Comptes Rendus Mathematique 338, 527 (2004), http://www.sciencedirect.com/science/article/pii/S1631073X04000780.

[19] N. R. Constable, D. Z. Freedman, M. Headrick, S. Minwalla, L. Motl, A. Postnikov and W. Skiba, "PP-wave string interactions from perturbative Yang-Mills theory", JHEP 0207, 017 (2002) hep-th/0205089.

[20] N. Beisert, C. Kristjansen, J. Plefka, G. W. Semenoff and M. Staudacher, "BMN Correlators and Operator Mixing in $\mathcal{N}=4$ Super Yang-Mills Theory", Nucl. Phys. B650, 125 (2003) hep-th/0208178

[21] N. R. Constable, D. Z. Freedman, M. Headrick and S. Minwalla, "Operator mixing and the BMN correspondence", JHEP 0210, 068 (2002), hep-th/0209002.

[22] B. Eden and A. Sfondrini, "Tessellating cushions: four-point functions in $\mathcal{N}=4$ SYM", JHEP 1710, 098 (2017), arxiv: 1611.05436

[23] T. Fleury and S. Komatsu, "Hexagonalization of Correlation Functions", JHEP 1701, 130 (2017), arxiv:1611.05577. 